DESY $18-045$

DO-TH 18/06

April 2018

\title{
Analytic Computing Methods for Precision Calculations in Quantum Field Theory
}

\author{
Johannes Blümlein $^{a}$ and Carsten Schneider ${ }^{b}$ \\ a Deutsches Elektronen-Synchrotron, DESY, \\ Platanenallee 6, D-15738 Zeuthen, Germany \\ ${ }^{b}$ Research Institute for Symbolic Computation (RISC), \\ Johannes Kepler University, Altenbergerstraße 69, A-4040, Linz, Austria
}

\begin{abstract}
An overview is presented on the current status of main mathematical computation methods for the multi-loop corrections to single scale observables in quantum field theory and the associated mathematical number and function spaces and algebras. At present massless single scale quantities can be calculated analytically in QCD to 4-loop order and single mass and double mass quantities to 3-loop order, while zero scale quantities have been calculated to 5-loop order. The precision requirements of the planned measurements, particularly at the FCC-ee, form important challenges to theory, and will need important extensions of the presently known methods.
\end{abstract}




\section{Introduction}

Precision calculations of radiative corrections in the Standard Model play an important role in determining its fundamental parameters, like the particle masses, coupling constants and mixing parameters and are important to reveal possible deviations due to new physics. These calculations are very challenging and have to be more precise than the experimental accuracy to be reached. At present the necessary calculations, both in the case of the physics at HERA and the LHC, for typical quantities, last about one to two decades until the final results are obtained. A similar and probably larger period is estimated to be necessary to meet the requirements at a future circular collider, such as the FCC [1]. This includes both the necessary development of new technologies, and to a lesser extent, also substantial computation times.

In this survey we will summarize the status reached in the field of analytic radiative corrections mostly for inclusive single and double scale quantities, widely concentrating on QED and QCD, and discuss the different techniques available at the higher loop level at present. Currently we are at the beginning of a discussion of what is needed at the theoretical side to meet the planned experimental accuracy for key processes, e.g. at the FCC-ee. This résumé of computational methods is meant to be one asset to explore in which way these technologies have to develop in the future, concerning the computational complexity and the different theoretical, mathematical and computer algebraic methods, as far as this is currently foreseeable.

The mathematical representation of the one-loop case has been given by 't Hooft and Veltman in [2-4] during the 1970ies and the analytic representation of 5- and higher point functions were given in [5-8] and references therein. Also the one-loop off-shell amplitudes are known in full detail [9-11]. At general space-time dimensions these quantities are represented in terms of higher hypergeometric functions, such as the hypergeometric function itself [12-14], Appell functions [15-21] and the Lauricella-Saran functions [21-24]. For the multi-leg corrections, containing more scales, mostly numerical methods have to be used at present, cf. [25] for a survey.

The most far reaching result on radiative corrections at LEP have been the complete $O\left(\alpha^{2}\right)$ QED corrections around the $Z$ resonance [26], cf. also [27]. During the 1990ies most of the single and some of the double scale two-loop corrections for different processes have been calculated in QCD, cf. e.g. [28-30]. At the mathematical side, polylogarithms [31,32] and Nielsen integrals [33-36] were used, leading to complicated argument structures.

Around $1998^{1}$ a more systematic account was needed to be able to perform more advanced calculations and to simplify what has been reached at the 2-loop level. This led to the introduction of the nested harmonic sums [38,39] and the harmonic polylogarithms [40]. Within this framework various important massless [41-44] and massive [45-47] 3-loop corrections could be calculated. In later computations extensions of these function spaces to generalized harmonic polylogarithms [48,49], cyclotomic harmonic sums and polylogarithms [50], and root-valued iterated integrals and finite weighted (inverse) binomial sums [51] were necessary. Finally, also elliptic integrals [52] appear in massive Feynman integral calculations [53-61], and modular forms and functions, see e.g. [62-64], play a role in describing the corresponding physical results. In this paper we will mainly discuss zero-, one-, and a few two-scale processes. In general, processes with more than two scales are important as well. However, the systematic mathematical study of the corresponding analytic master integrals, beyond the one-loop case, is at the beginning only.

The paper is organized as follows. In Section 2 we give an outline of a systematic way to classify Feynman integrals. It will be given studying the structure of the associated system of differential equations w.r.t. its degree of factorization. General computation methods are

\footnotetext{
${ }^{1}$ For computation techniques until 1998, see Ref. [37].
} 
summarized in Section 3. In Section 4 we describe a series of analytic integration methods for Feynman diagrams. There are universal corrections to scattering processes, characterized by logarithmic enhanced terms, which we describe in Section 5 using the structure function method. It relies on the renormalization group equation for factorization. The different kind of loop corrections can be presented by a series of function and special number spaces which will be reviewed in Section 6. Here we will mainly concentrate on the spaces for first-order factorizing systems. In Section 7 we will discuss non-first order factorizing systems and present an outlook in Section 8.

\section{A Systematic Way to Classify Feynman Integrals}

Feynman parameter integrals can be calculated directly by applying successive binomial

$$
\left(A\left(x_{i}\right)+B\left(x_{i}\right)\right)^{k}=\sum_{l=0}^{k}\left(\begin{array}{l}
k \\
l
\end{array}\right) A^{l}\left(x_{i}\right) B^{k-l}\left(x_{i}\right), k \in \mathbb{N},
$$

and Mellin-Barnes [65, 66]

$$
\frac{1}{\left(\left(A\left(x_{i}\right)+B\left(x_{i}\right)\right)^{\lambda}\right.}=\frac{1}{2 \pi i} \int_{-i \infty}^{+i \infty} d z \frac{B\left(x_{i}\right)^{z}}{A\left(x_{i}\right)^{\lambda+z}} \frac{\Gamma(\lambda+z) \Gamma(-z)}{\Gamma(\lambda)}, \lambda \in \mathbb{R}, \lambda>0,
$$

decompositions of the integrand implemented in different packages [67-69]. Here $\left\{x_{i}\right\}$ denotes the set of Feynman parameters and $A$ and $B$ are polynomials. These representations allow one to perform all Feynman parameter integrals. The remaining Mellin-Barnes integrals (2) have to be carried out using the residue theorem. In this way one obtains multi-sum representations, as has been outlined in Ref. [70]. However, the remaining summation problems may turn out to be very difficult to be solved using difference field and ring theory [71-83], algorithmically implemented in the package Sigma $[84,85]$. It may even turn out that the corresponding nested sums cannot be solved using difference field and ring theory in terms of indefinite nested sums defined over hypergeometric products because the associated difference equation does not factorize sufficiently.

It thus seems that there are apparently no general methods known a priori, to evaluate a given general Feynman diagram analytically. Therefore we want to attempt at least to find general classes first, for which the corresponding mathematical solution methods can be found or be developed, along with classifying the different degrees of complexity.

One way to proceed is the following. In most of the present and future Feynman diagram calculations one has to use the integration-by-part (IBP) method [86] to reduce the problem to master integrals. There are various implementations of Laporta's algorithm [87], as e.g. [88-90] and others. Given such a system, the following definition will be convenient for further considerations. An $n \times n$ system is said to decouple to $m$ th order, $m \leq n$, if the $n$ linear differential operators that contain the solutions of the corresponding unknown functions of the homogeneous system factorize to irreducible factors where the maximal order among these factors is $m$. Note that decoupling algorithms such as the cyclic-vector method provide such scalar equations for the unknown functions [91,92]. In our considerations below we will utilize Zürcher's algorithm [93] which might provide a refined version with several scalar equations. However, if the scalar equations found by any of the available decoupling algorithms factorize linearly, the system decouples to first-order, i.e., $m=1$. In particular, the system is completely solvable in terms of indefinite nested integrals (resp. indefinite nested sums in the recurrence case). This special case will be considered in detail in Section 4. 
In this way, one can determine the degree of uncoupling of the system and a systematic characteristics of sets of Feynman integrals can be given in terms of their master integrals. Here, the decoupling has been carried out in $x$-space, with $x$ being the variable of the differential equations. One may now consider a map to $N$-space either by a formal power series Ansatz or a Mellin transform and study the problem in terms of the corresponding system of difference equations. Usually the uncoupling can be different here. There are cases know in which, e.g., 1st order decoupling can be obtained in Mellin space [61] but not in $x$ space and vice versa [94]. If possible, one should always consider both cases. First order factorizing systems can be always solved in terms of iterative integrals or in $N$-space by indefinitely nested sums.

2nd order decoupling systems will have ${ }_{2} F_{1}$-solutions, with usually more than three singularities, cf. e.g. [54,61]. Much less is known about genuine 3rd and higher order systems, which are, however, expected to occur at higher loop order, or in the presence of more scales. The computational methods to solve 1st order systems, cf. Section 4, 6, are different from those of the 2nd order systems, cf. Section 7. It is therefore expected that at larger degrees of non-decoupling quite different mathematical methods are necessary for adequate analytic solutions of these radiative corrections, and may not even be completely known in the mathematical literature w.r.t. the function spaces covering the corresponding representation.

After a brief consideration of general computation methods for Feynman integrals, which are standard by now, in the next section, we turn to the case of 1st order factorization systems.

\section{General Computation Methods}

The present higher loop integrals make it necessary to evaluate a large number of Feynman integrals; their number will grow in future projects significantly. Currently there is no method known for the renormalizable quantum field theories realized in nature to start from any more compact structure. The generation of the Feynman diagrams, using packages like qgraf [95], still seems to be possible as well as the calculation of the corresponding color structures using Color [96]. Furthermore, standardized algorithms to obtain Feynman parameterizations exist, cf. e.g. [97-99]. However, with growing complexity, to perform the Dirac- and spin-algebra will be a challenge even to FORM [100-103]. These packages need steady maintenance and will need further refinements. It will be indispensable to reduce the corresponding scalar (or tensor) integrals to master integrals. Here, at present, we have a series of efficient algorithms like Air, FIRE, Crusher, REDUZE [88-90, 104, 105]. These algorithms definitely will need further extensions and improvements to face future problems. Before being able to embark to the analytic calculation of master integrals one has to have numerical tools of sufficient accuracy to evaluate the master integrals setting their remaining parameters to specific values. Here the method of sector decomposition and related numerical methods are used, see e.g. [106-117]. These methods have also to be refined further to meet future tasks.

\section{A Series of Analytic Integration Methods}

We turn now to the analytic integration of Feynman integrals and master integrals, respectively. In the following, we limit the consideration mainly to zero- and single scale processes. Most of the methods lead to representations of nested finite and infinite sums, which have to be solved applying summation techniques. These are therefore a central tool in the calculation of Feynman integrals. Non of the methods, which we are going to discuss in the following, has a thorough superiority over an other in case it can be applied. As experience in larger projects shows, one 
method is better suited for the calculation of certain diagrams than others. The differences in run-time can sometimes be quite large and e.g. amount to different weeks compared to seconds. Let us now turn to a series of specific integration methods.

\subsection{The PSLQ method}

The PSLQ method [118] is an integer algorithm with which a possible dependence between certain constants, expressed by floating point numbers to a certain accuracy, can be determined. To do this one starts with a certain accuracy of $n$ digits and tries to find a first relation, usually given by a sequence of rational numbers, with a certain denominator size. One then increases the accuracy and checks whether the relation is found again. The method has no proof character, however, it is able to establish approximate relations between constants at a high accuracy. If a correct relation is found, one can proof it by rigorous methods later, which, however, is usually by far more demanding. The well-know relation

$$
\operatorname{Li}_{3}\left(\frac{1}{2}\right)=\frac{7}{8} \zeta_{3}-\frac{1}{2} \zeta_{2} \ln (2)+\frac{1}{6} \ln ^{3}(2)
$$

can be found already providing 9 digits. In this case one knows, that all participating numbers in the r.h.s. are irrational. Usually one needs many more digits, however, cf. e.g. [119, 120].

The method can be very efficiently used if one knows the set of numbers, by which the result of a calculation is finally spanned. In intermediary steps many more unknown constants may appear, which cancel in the final result. This has been very impressively demonstrated in the calculation of the 5-loop $\beta$-function in QCD in Ref. [121], where for the master integrals even large amounts of elliptic and probably trans-elliptic constants contribute, which all cancel finally. It was expected, that the 5 -loop $\beta$-function depends on $\zeta$-values [122] only and then also known by the results of Refs. [123, 124].

Similarly, one makes use of this also in the method of arbitrarily high moments, Section 4.8.

E.g. for the calculation of the massive 3-loop operator matrix element $(\mathrm{OME}) A_{Q g}^{(3)}(N)$, it is known, that their moments depend on a series of $\zeta$-values only, [125]. The master integrals and the general $N$-result contain elliptic integrals [61], on the other hand.

The PSLQ-method provides a great heuristic algorithm to potentially simplify zero-scale quantities in higher order loop calculations.

\subsection{Guessing difference equations}

Often it is easier to calculate a large series of zero-scale quantities, e.g. the Mellin moments,

$$
G(N)=\mathbf{M}[f(x)](N)=\int_{0}^{1} d x x^{N-1} f(x),
$$

of a sought single-scale quantity $G(N)$ for general values of $N$. One therefore seeks ways to find the associated difference equation [126] to the set of moments $\{G(2), G(4), \ldots, G(2 m)\}, m \in \mathbb{N}$ [127-130]. The reason why one may hope to find a difference equation is that $G(N)$ is a function obeying a recurrence. This is the case for many objects in quantum field theory, as e.g. (massive) operator matrix elements [125], but also single-scale Wilson coefficients, Ref. [43].

A possible algorithm to calculate the string of moments $\left\{\left.G(k)\right|_{k=2} ^{2 m}\right\}$ as input is described in Section 4.8. One then applies the guessing algorithm [131], now also available in Sage [132], exploiting the fast integer algorithms available there. At a critical number $m$ a conjectured 
difference equation is obtained, the validity of which can be tested by shifting to a further number of higher moments. This method has been applied in Ref. [133] to obtain from more than 5000 moments the massless unpolarized 3-loop anomalous dimensions and Wilson coefficients in deepinelastic scattering [41-43]. Recently, the method has been applied ab initio in the calculation of 3-loop splitting functions [44] and the massive 2- and 3-loop form factor [44,134]. In the case of a massive operator matrix element 8000 moments [135] could be calculated and difference equations were derived for all contributing color and $\zeta$-value structures. Using the summation methods implemented in the package Sigma [84,85] one can solve these difference equations, whenever they are first order factorizing, or separate their first order factorizing parts, in case they are not, cf. Section 4.9. In Section 4.8 we will describe in which way the corresponding input-moments can be obtained.

\subsection{Generalized hypergeometric functions}

In calculating simpler Feynman integrals, or those with a partial factorization to simpler structures, the Feynman parameter representations exhibit integrand structures of Euler Betafunctions, the hypergeometric functions or their generalization [12-14] in general space-time dimensions, e.g. $D=4+\varepsilon$. Up to the level of massless and single mass two-loop integrals, cf. [28, 30, 136, 137], these representations are usually sufficient.

There is a hierarchy of ${ }_{p+1} F_{p}$ functions, the first of which read

$$
\begin{aligned}
B\left(a_{1}, a_{2}\right) & =\int_{0}^{1} d t t^{a_{1}-1}(1-t)^{a_{2}-1} \\
{ }_{2} F_{1}\left(a_{1}, a_{2}, b_{1} ; x\right) & =\frac{\Gamma\left(b_{1}\right)}{\Gamma\left(a_{2}\right) \Gamma\left(b_{1}-a_{2}\right)} \int_{0}^{1} d t t^{a_{2}-1}(1-t)^{b_{1}-a_{2}-1}(1-t x)^{-a_{1}} \\
{ }_{3} F_{2}\left(a_{1}, a_{2}, b_{1} ; x\right) & =\frac{\Gamma\left(b_{2}\right)}{\Gamma\left(a_{3}\right) \Gamma\left(b_{2}-a_{3}\right)} \int_{0}^{1} d t t^{a_{3}-1}(1-t)^{-a_{3}+b_{2}-1}{ }_{2} F_{1}\left(a_{1}, a_{2}, b_{1} ; t x\right) .
\end{aligned}
$$

Here the parameters $a_{i}, b_{i}$ are such, that the corresponding integrals exists, [138]. At 3-loop order, there are topologies which can be well described by Appell functions [15-21, 139, 140], cf. [141,142], e.g. the Appell $F_{1}$ function. It has the integral representation

$$
\begin{array}{r}
F_{1}\left(a, b_{1}, b_{2}, c ; x, y\right)=\frac{\Gamma(c)}{\Gamma(a) \Gamma(c-a)} \int_{0}^{1} d t t^{a-1}(1-t)^{c-a-1}(1-x t)^{-b_{1}}(1-y t)^{-b_{2}}, \\
\operatorname{Re}(c)>\operatorname{Re}(a)>0 .
\end{array}
$$

All the ${ }_{p+1} F_{p}$ functions have a single infinite sum representation, while the Appell-functions are represented by two infinite sums. Meeting these structures a larger number of integrals is mapped to these remaining sums only. In the physical applications the parameters $a_{i}, b_{i}, c_{i}, \ldots$ are functions of the dimensional parameter $\varepsilon$, in which these quantities have to be expanded. One finally obtains infinite sum representations, which have to be calculated using general summation methods, cf. Section 4.9. There are also some other classes of named higher transcendental functions, which obey multi-sum representations [18, 19,21]. For more complicated integrandstructures, however, one has to apply other techniques of integration, to which we turn now.

\subsection{Mellin-Barnes integrals}

All the higher transcendental functions being discussed in Section 4.3 have representations in terms of Pochhammer-Umlauf integrals, see [12], and also by Mellin-Barnes integrals. It provides 
also the natural generalization for cases in which the representation in terms of special functions is not known. Feynman parameter integrals usually exhibit denominators which are hyperexponential, i.e. are products of factors $\left(P_{j}\left(x_{i}\right)\right)^{\lambda_{j}}, \lambda_{j} \in \mathbb{R}, \lambda_{j}>0$. One may apply the MellinBarnes decomposition (2) to them. Here one may use the packages and methods described in [67-69, 143-145]. As outlined above, one obtains nested infinite sum representations after applying the residue theorem. These sums have to be solved by applying summation techniques, see Section 4.9. On the other hand, one may obtain numerical results also in performing the Mellin-Barnes integrals numerically, cf. e.g. [69,143-145], which provides useful checks. In aiming at an analytic result one should introduce Mellin-Barnes representations as little and as careful as possible, to avoid to shift the solution of the problem widely to the summation techniques.

\subsection{The method of hyperlogarithms}

If a Feynman diagram has no pole terms in the dimensional parameter $\varepsilon$ or can be made finite by certain transformations splitting off its pole terms [146], it can be calculated under certain conditions using the method of hyperlogarithms [147]. Since here the denominator of the Feynman integral is a multinomial in the Feynman parameters $x_{i} \in[0,1]$ one may seek a sequence of integrations, such that the denominator always is a linear function (Fubini sequence) in the integration variable under consideration. In this case the Feynman integral can be found as a linear combination of Kummer-Poincaré iterated integrals, see Section 6.3. The method has been first devised for massless scalar integrals in [147], for a corresponding code see [148]. The method has been generalized to massive Feynman diagrams, also containing local operator insertions in Ref. [149], extending its validity even to cases with no thorough multi-linearity. An implementation can be found in [150].

\subsection{The Almkvist-Zeilberger Algorithm}

Feynman parameter integrals usually appear as multi-integrals over $\left\{\left.x_{i}\right|_{1=1} ^{n}\right\} \in[0,1]^{n}$. Furthermore, in the single-variate case, they depend on a real variable $x$ as $I(x)$ or an integer variable $N$ as $I(N)$, and usually will depend in addition on the dimensional parameter $\varepsilon$. One is now interested in finding either an associated differential equation for $I(x)$ or a difference equation for $I(N)$. The Almkvist-Zeilberger algorithm [151,152] allows to find these equations which are of the form

$$
\begin{aligned}
\sum_{l=0}^{m} P_{l}(x, \varepsilon) \frac{d^{l}}{d x^{l}} I(x, \varepsilon) & =N(x, \varepsilon) \\
\sum_{l=0}^{m} R_{l}(N, \varepsilon) I(N+l, \varepsilon) & =M(N, \varepsilon),
\end{aligned}
$$

where $P_{l}, R_{l}$ are polynomials and $N(x, \varepsilon), M(N, \varepsilon)$ denote potential inhomogeneities. Internally, the equations $(9,10)$ are found by solving an underlying linear system of equations. The algorithm transforms an $n$-fold integration problem into a single equation, which can then be solved, by applying the techniques described in the subsequent sections. The bounds on $m$, given in the literature, are usually much higher, than what is found in praxis. An optimized and improved algorithm for the input class of Feynman integrals has been implemented in the Multi Integrate package $[142,153]$. It can either produce equations of the form $(9),(10)$ where the right hand sides are zero, or it can provide inhomogeneous equations with further tactics to simplify the inhomogeneous sides using tools introduced in the next subsections. 


\subsection{Differential equations}

The reduction of Feynman integrals to master integrals by the IBP-relations may also be used to obtain a system of ordinary differential equations in one of the parameters of the master integrals, or a system of partial differential equations in the case of more parameters. The master integrals may then be obtained as the solution of these systems under appropriate boundary conditions, [154-157]. For single-variate systems one considers

$$
\frac{d}{d x}\left(\begin{array}{c}
f_{1} \\
\vdots \\
f_{n}
\end{array}\right)=\left(\begin{array}{ccc}
A_{11} & \ldots & A_{1, n} \\
\vdots & & \vdots \\
A_{n 1} & \ldots & A_{n, n}
\end{array}\right)\left(\begin{array}{c}
f_{1} \\
\vdots \\
f_{n}
\end{array}\right)+\left(\begin{array}{c}
g_{1} \\
\vdots \\
g_{n}
\end{array}\right)
$$

which may be transformed into the scalar differential equation

$$
\sum_{k=0}^{n} p_{n-k}(x) \frac{d^{n-k}}{d x^{n-k}} f_{1}(x)=\bar{g}(x),
$$

with $p_{n} \neq 0$, and $(n-1)$ equations for the remaining solutions, which are fully determined by the solution $f_{1}(x)$.

An important class is formed by the 1st order factorizing systems, cf. Section 2, after applying the decoupling methods [93,158] encoded in Oresys [91]. They appear as the most simple case. One may transform the decoupled system into Mellin space and use the efficient solvers of the package Sigma there, cf. Ref. [142] and Section 4.9.

The decoupled differential operator of (12) can be written in form of a non-commutative product of first order differential operators and rational terms, which can now be integrated directly. The result is given by iterative integrals, also using partial fractioning and integration by parts to obtain representations in terms of proper letters,

$$
\begin{aligned}
& f_{1}(x)=\sum_{k=1}^{n+1} \gamma_{k} g_{k}(x), \gamma_{k} \in \mathbb{C} \\
& g_{k}(x)=h_{0}(x) \int_{0}^{x} d y_{1} h_{1}\left(y_{1}\right) \int_{0}^{y_{1}} d y_{2} h_{2}\left(y_{2}\right) \ldots \int_{0}^{y_{k-2}} d y_{k-1} h_{k-1}\left(y_{k-1}\right) \int_{0}^{y_{k-1}} d y_{k} q_{k}\left(y_{k}\right)
\end{aligned}
$$

with $q_{k}(x)=0$ for $1 \leq k \leq m$. Further, $\gamma_{m+1}=0$ if $\bar{g}(x)=0$ in $(12)$, and $\gamma_{m+1}=1$ and $q_{m+1}(x)$ being a mild variation of $\bar{g}(x)$ if $\bar{g}(x) \neq 0$. Note that these solutions are also called d'Alembertian solutions [159]. As the master integrals appearing in quantum field theories obey differential equations with rational coefficients, the letters $h_{i}$, which constitute the iterative integrals, have to be algebraic, see Section 6. Given a differential equation (12), the d'Alembertian solutions (13) can be computed with the package HarmonicSums [160]. More generally, also Liouvillian solutions [161] can be calculated with HarmonicSums utilizing Kovacic's algorithm [162]; this algorithms finds also Liouvillian solutions of second order differential equations. Summarizing, representation (13) can be found in the 1st order decoupling case of a coupled system of the form (11) for any basis of master integrals, cf. [142] $]^{2}$. If being transformed to the associated system of difference equations, the same holds, if this system is also first order factorizing. The solution of the remaining equations are directly obtained by the first solution.

The corresponding algorithms hold to all orders in $\varepsilon$, on the expense that it is necessary to obtain higher order expansions to cancel the respective poles in the expansion of the coefficients.

\footnotetext{
${ }^{2}$ This algorithm has been applied in many massive 3-loop calculations so far, cf. [135] for a survey, and also in [163].
} 
This algorithm has some relation to the method of hyperlogarithms, cf. Section 4.5. For an implementation see Ref. [134]. For the solution of 1st order decoupling systems also algorithms given in [164] are useful.

In the multi-variate case, the $\varepsilon$-representation of a linear system of partial differential equations

$$
\partial_{m} f\left(\varepsilon, x_{n}\right)=A_{m}\left(\varepsilon, x_{n}\right) f\left(\varepsilon, x_{n}\right)
$$

is important, as has been recognized in Refs. [165,166], see also [167]. One considers the wellknown zero-curvature representations [168, 169]

$$
\partial_{n} A_{m}-\partial_{m} A_{n}+\left[A_{n}, A_{m}\right]=0,
$$

with $[A, B]$ a Lie bracket [170]. The matrices $A_{n}$ can now be transformed (in the general nonAbelian case) by

$$
A_{m}^{\prime}=B^{-1} A_{m} B-B^{-1}\left(\partial_{m} B\right)
$$

see also $[168,169]$. One now intends to find a matrix $B$ to transform $(15)$ into the form

$$
\partial_{m} f\left(\varepsilon, x_{n}\right)=\varepsilon A_{m}\left(x_{n}\right) f\left(\varepsilon, x_{n}\right)
$$

if possible. This then allows solutions in terms of iterative integrals. The corresponding system (15), therefore, has also to belong to the first order factorizing case. A formalism for the basis change to the $\varepsilon$-basis has been proposed in [171] and implemented in the single-variate case in $[172,173]$ and in the multi-variate case in [174]. In this description one has the advantage to work at equal weight, which avoids necessary deeper expansions in $\varepsilon$ needed otherwise.

\subsection{The method of arbitrarily high moments}

Standard procedures like Mincer [175] $]^{3}$, MATAD [180] or Q2E [180,181] allow the calculation of a comparable small number of Mellin moments of a given quantity only, because the resources needed by these algorithms grow exponentially. Alternatively, one may think of using difference equations for the master integrals of a physical problem in Mellin space to calculate the integer moments of the physical quantity studied to obtain higher and higher moments. This has been pursued in Ref. [182] and the formalism also applies when only ordinary differential equations are available for the master integrals. Furthermore, here we need not to know the degree of decoupling of the corresponding system, which can be rather high.

The most extensive system having been studied using this method so far led to 8000 moments [135] after several weeks of computation time. In applications the structure of the Mellin moments is usually mathematically much simpler than the corresponding general expressions in $x$ and $N$ space. They are given by rational numbers and are labeled by special constants, like MZVs. Furthermore, the physical quantity to be calculated can turn out to be structurally less complex than the master integrals. Moreover, unlike the latter, one has to calculate it only to $O\left(\varepsilon^{0}\right)$.

Finally, one projects onto color factors and special constants to obtain rational sequences. They are used as input to guessing to seek for a closed form difference equation in all cases. We could achieve this also in all the complex cases mentioned. The final step consists now

\footnotetext{
${ }^{3}$ See also $[176,177]$ for Baikov's method, which allowed a large number of calculations up to the 5-loop level as e.g. [123]. For the solution of important 4-loop problems Forcer [178] and the $R^{*}$ [179] method have been developed.
} 
in solving these difference equations using the methods of the package Sigma. For a series of difference equations a solution has been found. In others only the 1st order factorizing factors were obtained, leaving remainder 4th order systems, which are further investigated using the methods described in Section 7. Here the last system decoupled into two $2 \times 2$ systems.

The method of arbitrarily high moments is a rather global one. E.g. in 1st order factorizable systems like they appear in massless calculations, e.g. for the 3 loop anomalous dimensions [44] and related quantities, the method is fully automated.

\subsection{Summation Techniques in $\Pi \Sigma$-Fields and Rings}

As described in Sections 4.3 and 4.6, the evaluation of Feynman integrals can be often reduced to the problem to simplify multiple sums defined over hypergeometric products, harmonic numbers and generalizations thereof $[39,49-51,158,183]$; see also Section 6. Given such a sum representation, symbolic summation algorithms in the setting of difference rings and fields [71-83] can be utilized to derive an alternative representation in terms of indefinite nested sums defined over hypergeometric products. Special cases of these sums are enumerated in Section 6. More precisely, using the Mathematica package Sigma $[84,85]$ the following three steps lead often to the desired simplification: (1) compute a linear recurrence relation for the sum using the creative telescoping paradigm [184] in the setting of difference rings, and (2) solve afterwards the recurrence in terms of d'Alembertian solutions [159,185]. This means that one finds all solutions that can be given in terms indefinite nested sums defined over hypergeometric products. Note that all the sums from Section 6 are covered. Finally, in step (3) one tries to combine the solutions to derive a simpler representation of the original input sum. The package EvaluateMultiSums [186, 187] based on Sigma combines all these steps and variants thereof in a very efficient way to carry out such simplifications automatically. Here one works from inside to outside: one considers first the innermost definite sum and transforms it with the above method to an expression in terms of indefinite nested sums. Afterwards, one treats the next definite summation quantifier and transforms this sum to an expression in terms of indefinite nested sums, etc.

A crucial step for the above approach is that in a preprocessing step one first gets rid of the dimensional parameter $\varepsilon$ by expanding first the summand of the multiple sum w.r.t. $\varepsilon$, i.e., computes the first coefficients of its Laurent series expansion and by applying afterwards the summation quantifiers to each of the coefficients. Only then the summation technologies described above are applied to the summations of the coefficients up to the desired order. Alternatively, there are algorithms available that can compute directly for a multiple sum a recurrence of the form (10) depending also on $\varepsilon$. In particular, if the summand consists of a product of hypergeometric products, one can utilize WZ-summation theory [188]; for refined methods and an efficient implementation we refer to the MultiSum package [189]. This approach can be considered as the discrete version of the Almkvist-Zeilberger algorithm introduced in Section 4.6. Note that one can use also holonomic summation algorithms [190,191] to derive recurrences of the form (10); for a generalized version combining the holonomic and difference ring approach we refer to [192] and references therein. Finally, one can use Sigma's recurrence solver described in [70]. Suppose that one is given a recurrence of the form (10) where the inhomogeneous part is given as an $\varepsilon$-expansion; more precisely, the first coefficients are given in terms of indefinite nested sums defined over hypergeometric products. Then together with the first initial values of the Feynman integral $I(N)$, which is a solution of (10), one can decide constructively if the coefficients of the $\varepsilon$-expansion of $I(N)$ can be again given in terms of indefinite nested sums defined over hypergeometric products. Note that Sigma's recurrence solver can be also utilized to solve recurrences coming, e.g., from the Almkvist-Zeilberger algorithm; see Section 4.6. 
In many applications, the transformation of Feynman integrals yields an enormous expression consisting of up to thousands of multiple sums. To simplify these sums successively using these summation tools is not only problematic because of time restrictions, but also because of the following intrinsic problem: The scattered sums themselves cannot be simplified in terms of indefinite nested sums, but only a suitable combination of them can be simplified in this way. In order to bypass these problems, the package SumProduction $[187,193]$ built on Sigma can be utilized: it reduces the sum expressions to compact forms where the arising sums are merged appropriately. Afterwards EvaluateMultiSum can be applied to this optimized expression within reasonable time and without dealing with sums that cannot be handled within the difference ring setting.

Besides the simplification of multiple sums, the difference ring approach can be also used to solve coupled systems of differential and difference equations [142, 194, 195]. More precisely, given a finite number of Feynman integrals $F_{1}(N), \ldots, F_{n}(N)$ depending on a Mellin variable $N$ which are determined by a system of linear difference equations equipped with sufficiently many initial conditions, the package SolveCoupledSystem decides algorithmically if the Feynman integrals can be expressed in terms of indefinite nested sums defined over hypergeometric products. Internally, the following machinery is applied. (1) Zürcher's algorithm [93] implemented in the package OreSys [91] is used to decouple the system to one scalar recurrence that characterizes one of the given Feynman integrals, say $F_{1}(N)$, together with explicitly given linear combinations of $F_{1}(N)$ (and possible shifts) describing the remaining integrals $F_{2}(N), \ldots, F_{n}(N)$ (sometimes one obtains several scalar recurrences for a subset of the given integrals). (2) Then using Sigma's recurrence solver one can decide constructively if the Feynman integral $F_{1}(N)$ (or the coefficients of the $\varepsilon$-expansion of $\left.F_{1}(N)\right)$ can be written in terms of indefinite nested sums. If this is not possible, it follows that also $F_{2}(N), \ldots, F_{n}(N)$ cannot be expressed in terms of indefinite nested sums. However, if it is possible, also $F_{2}(N), \ldots, F_{n}(N)$ can be assembled to expressions in terms of indefinite nested sums using the linear combination of $F_{1}(N)$ (and its shifted versions).

In addition, this method can be generalized to solve coupled systems of differential equations coming from IBP methods [86-90]; see also Sections 2, 4.7. Here one assumes that the Feynman integrals $f_{1}(x), \ldots, f_{n}(x)$ can be given in the power series representations

$$
f_{i}(x)=\sum_{N=0}^{\infty} F_{i}(N) x^{N}
$$

for $i=1, \ldots, n$. Then given a coupled system of linear differential equations of the form ${ }^{4}(11)$ in the $f_{1}(x), \ldots, f_{n}(x)$, one can decide constructively, if the coefficients $F_{1}(N), \ldots, F_{n}(N)$ (or the coefficients of their $\varepsilon$-expansions) can be represented in terms of indefinite nested sums. Internally, this problem can be reduced to the problem to solve a coupled system of difference equations as follows. Comparing coefficients w.r.t. $x^{N}$, the coupled system of differential equations in the $f_{1}(x), \ldots, f_{n}(x)$ yield a coupled system of linear difference equations in the $F_{1}(N), \ldots, F_{n}(N)$, and afterwards the above algorithm is applied; compare [142,194,195]. Alternatively, one can first decouple the coupled system of linear differential equations, afterwards transforms the scalar differential equation to a scalar recurrence by comparing coefficients w.r.t. $x^{N}$, and finally one applies Sigma to solve the scalar recurrence. For further details we refer to [196].

\footnotetext{
${ }^{4}$ In addition, we assume that the the inhomogeneous parts $g_{i}(x)$ in $(11)$ are given in power series representations were the coefficients can be written in terms of indefinite nested sums defined over hypergeometric products.
} 


\section{Factorizing Corrections: The Structure Function Method}

In the case of radiation off (meta)stable massive fermions, like the charged leptons and heavy quarks of mass $m$, the logarithmically enhanced contributions can be determined by the renormalization group equations $[197,198]$ describing the factorization of the process of the corresponding scattering cross section

$$
\sigma(s)=C\left(\frac{s}{\mu^{2}}\right) \otimes A\left(\frac{\mu^{2}}{m^{2}}\right),
$$

neglecting power corrections. Here $s$ denotes the CMS energy squared and $\otimes$ the Mellin convolution

$$
A(x) \otimes B(x)=\int_{0}^{1} d x_{1} \int_{0}^{1} d x_{2} \delta\left(x-x_{1} x_{2}\right) A\left(x_{1}\right) B\left(x_{2}\right) .
$$

One calls the corrections due to the function $A(x)$ factorizing, because of its connection to $C(x)$ by a Mellin convolution, which factorizes, if considered in Mellin space, via

$$
\mathbf{M}[A(x) \otimes C(x)](N)=\mathbf{M}[A(x)](N) \cdot \mathbf{M}[C(x)](N) .
$$

Both the coefficient function $C(x)$ and the operator matrix elements $A(x)$ obey a series expansion in the coupling constant $a=\alpha /(4 \pi)$,

$$
\begin{aligned}
A(x) & =\sum_{k=0}^{\infty} a^{k} \sum_{l=0}^{k} A_{k, l}(x) \ln ^{l}\left(\frac{\mu^{2}}{m^{2}}\right) \\
C(x) & =\delta(1-x)+\sum_{k=1}^{\infty} a^{k} \sum_{l=0}^{k} C_{k, l}(x) \ln ^{l}\left(\frac{s}{\mu^{2}}\right)
\end{aligned}
$$

such that in the expansion of $\sigma(s)$ the dependence on the factorization scale $\mu$ vanishes and all logarithmic terms depend on the ratio $s / m^{2}$ only, see e.g. [199]. Here the expansion coefficients of the coefficient function $C_{k, l}$ are process dependent, while the ones of the operator matrix elements $A_{k, l}$ are process independent. Since the decomposition (19) has been studied for deep-inelastic structure functions first one calls this representation also the structure function method.

Let us consider the QED corrections in the inclusive case as an example. The leading order corrections are of $O\left(\left(a \ln \left(s / m^{2}\right)\right)^{k}\right)$. These are universal. For the QED corrections, initial- and final state corrections have been calculated to various processes, including light fermion emission, in Refs. [200-206] up to $O\left(\left(a \ln \left(s / m^{2}\right)\right)^{5}\right)$ in the unpolarized and polarized case. One may as well treat sub-leading logarithms, as lined out in Refs. [26,27,207], see also [208]. These corrections are no longer universal, but depend on the different contributing subprocesses of the observable considered. The structure function method only applies to situations in which power corrections of $O\left(\mathrm{~m}^{2} / \mathrm{s}\right)$ can be safely neglected.

\section{Function Spaces for First Order Factorizing Systems}

When solving first order factorizing systems it is not known a priori, which particular alphabet will span the function (distribution) space of the solutions. All what is known is the structure of the coefficients of the respective determining differential or difference equation and that the 
letters of the alphabet will result from them. In Section 4 we have outlined a series of solution algorithms which finally also determine the corresponding alphabets. In the following we will concentrate on the cases which have been found in quantum field theory so far. Usually the letters appearing in the different subsets may even appear combined in applications ${ }^{5}$. All the functions described in the following and their mutual relations are implemented in the package HarmonicSums [38, 39, 49-51, 153, 160, 209, 210].

\subsection{Harmonic sums and harmonic polylogarithms}

Next to purely rational functions in either the Mellin variable $N$ or the momentum-fraction variable $x \in[0,1]$, one obtains the nested harmonic sums [38,39]

$$
S_{b, \vec{a}}(N)=\sum_{k=1}^{N} \frac{(\operatorname{sign}(b))^{k}}{k^{|b|}} S_{\vec{a}}(k), \quad a_{i}, b, N \in \mathbb{N} \backslash\{0\}, \quad S_{\emptyset}=1,
$$

and the harmonic polylogarithms [40]

$$
H_{b, \vec{a}}(x)=\int_{0}^{x} d y f_{b}(y) H_{\vec{a}}(y), \quad b, a_{i} \in\{-1,0,1\}, \quad H_{\emptyset}(x)=1,
$$

with the letters

$$
f_{-1}(x)=\frac{1}{1+x}, \quad f_{0}(x)=\frac{1}{x}, \quad f_{1}(x)=\frac{1}{1-x} .
$$

There are subsets of (24), like the non-alternating harmonic sums with $a_{i}, b>0$, and of (25) with the alphabets $\{0,1\}$ and $\{0,-1\}$, the Nielsen integrals [33-36]. A subset of the latter are the canonical polylogarithms $[31,32] .{ }^{6}$

Both function spaces form shuffle algebras in the case of the functions, cf. [212, 213], and quasi-shuffle algebras in case of the sums, cf. [212-214] $]^{7}$,

$$
\begin{aligned}
S_{a}(N) & \uplus S_{b_{1}, \ldots, b_{m}}(N)=S_{a, b_{1}, \ldots, b_{m}}(N)+S_{b_{1}, a, \ldots, b_{m}}(N)+\ldots+S_{b_{1}, \ldots, b_{m}, a}(N) \\
H_{a}(x) \cdot H_{b_{1}, \ldots, b_{m}}(x) & =H_{a}(x) ш H_{b_{1}, \ldots, b_{m}}(x)=H_{a, b_{1}, \ldots, b_{m}}(x)+H_{b_{1}, a, \ldots, b_{m}}(x)+\ldots+H_{b_{1}, \ldots, b_{m}, a}(x)
\end{aligned}
$$

and form Hopf-algebras [215-217], which also form the basis of renormalizable quantum field theories [218-221]. The bases of these algebras can be counted using Lyndon words [222, 223] and by using Witt formulae [224,225]. Sum products obey quasi-shuffle relations as

$$
\begin{aligned}
S_{a}(N) \cdot S_{b_{1}, \ldots, b_{m}}(N)= & S_{a}(N) ш S_{b_{1}, \ldots, b_{m}}(N)+S_{a_{1} \wedge b_{1}, b_{2}, \ldots, b_{m}}(N)+S_{b_{1}, a_{1} \wedge b_{2}, b_{3}, \ldots, b_{m}}(N) \\
& +\ldots+S_{b_{1}, \ldots, b_{m-1}, a_{1} \wedge b_{m}}(N),
\end{aligned}
$$

with $a \wedge b=\operatorname{sign}(a) \operatorname{sign}(b)(|a|+|b|)$. It has been shown in [226] that the existing relations given in the quasi-shuffle algebra coincide with the relations in the ring of sequences when one evaluates

\footnotetext{
${ }^{5}$ For previous surveys see Refs. [158,183].

${ }^{6}$ Some integration methods may lead to very sophisticated argument structures of polylogarithms, harmonic polylogarithms and related functions. The method of the symbol [211] can be used to simplify, and to essentially compactify the corresponding expressions.

${ }^{7}$ The property to form a shuffle algebra is quite general and it will apply also to the other function spaces discussed below.
} 
the elements to sequences. To both spaces there is a single space of special constants associated, which is obtained in the limit $N \rightarrow \infty$ in case of the sums and $x \rightarrow 1$, provided the limits exist. These are the multiple zeta values (MZVs) [122]. There are more relations between these constants, than between the nested sums and the iterated integrals. For the alphabets $\{0,1\}$ the relations were given up to $\mathrm{w}=22$ and for $\{-1,0,1\}$ to $\mathrm{w}=12$, respectively, in Ref. [122].

One may add the symbol $\sigma_{0}:=\sum_{k=1}^{\infty}(1 / k)$ to the set formally, expressing the respective degree of divergence. For the set $\{-1,0,1\}$ the basic constants through polynomials of which all other constants can be obtained are

$$
\left\{\sigma_{0}, \ln (2) ; \zeta_{2} ; \zeta_{3} ; \operatorname{Li}_{4}\left(\frac{1}{2}\right) ; \zeta_{5}, \operatorname{Li}_{5}\left(\frac{1}{2}\right) ; \operatorname{Li}_{6}\left(\frac{1}{2}\right) ; \zeta_{7}, \operatorname{Li}_{7}\left(\frac{1}{2}\right), \sigma_{-5,1,1}, \sigma_{5,-1,-1} ; \ldots\right\}
$$

from weight $w=1$ to 7 . Here

$$
\zeta_{k}=\sum_{l=1}^{\infty} \frac{1}{l^{k}}, \quad k \in \mathbb{N}, k \geq 2 ; \quad \operatorname{Li}_{k}(x)=\sum_{l=1}^{\infty} \frac{x^{l}}{l^{k}},|x| \leq 1 ; \quad \sigma_{a, b, c}=\lim _{N \rightarrow \infty} S_{a, b, c}(N) .
$$

The Mellin transform maps the elements of both spaces, i.e. the Mellin transform of a harmonic polylogarithm can be represented by a linear combination of nested harmonic sums and their values in the limit $N \rightarrow \infty$, e.g.

$$
\mathbf{M}\left[H_{0,1,1}(x)\right](N)=-\frac{S_{1,1}(N)}{N^{2}}+\frac{\zeta_{3}}{N} .
$$

Applying the quasi-shuffle relations one may further reduce

$$
S_{1,1}(N)=\frac{1}{2}\left[S_{1}^{2}(N)+S_{2}(N)\right] .
$$

The harmonic sums can be analytically continued from $N \in \mathbb{N}$ to $N \in \mathbb{C}$ for even resp. odd moments using their representation in terms of Mellin transforms. The latter are expanded into factorial series $[227,228]$ and factors of $S_{1, \ldots, 1}(N)$, given by polynomials of poly-gamma functions and MZVs. Due to this one may differentiate harmonic sums for $N$ and derive besides the quasi-shuffle relations structural relations [229,230]. Approximate numerical representations for the analytic continuation of harmonic sums have also been obtained [231-233]. It finally can be shown that the inclusive 2-loop results in the massless and single-mass case, neglecting power corrections, can be expressed by only six harmonic sums [234,235]

$$
S_{1}(N), S_{2,1}(N), S_{-2,1}(N), S_{-3,1}(N), S_{2,1,1}(N), S_{-2,1,1}(N)
$$

see also $[229,230]$. Harmonic sums and polylogarithms are also implemented in the packages summer [38], harmpol [40] and HPL [236]. Precise numerical representations of harmonic sums were given in [237, 238].

\subsection{Cyclotomic harmonic sums and harmonic polylogarithms}

An extension of the harmonic sums and polylogarithms are the cyclotomic harmonic sums and harmonic polylogarithms, the properties of which have been studied in Ref. [50]. The cyclotomic harmonic polylogarithms are the iterated integrals over the alphabet

$$
\left\{\frac{1}{x},\left.\frac{x^{l}}{\Phi_{k}(x)}\right|_{0 \leq l \leq \varphi(k)}\right\}
$$


with $\Phi_{k}(x)$ the $k$ th cyclotomic polynomial consecutively found by factorizing $\left(x^{N}-1\right)$ and $\varphi(k)$ the totient function. Iterated integrals of these letters are called cyclotomic harmonic polylogarithms, where $\Phi_{1}(x)=1 /(1-x), \Phi_{2}(x)=1 /(1+x), \Phi_{3}(x)=1 /\left(1+x+x^{2}\right), \Phi_{4}(x)=$ $1 /\left(1+x^{2}\right)$ and $\Phi_{6}(x)=1 /\left(1-x+x^{2}\right)$. The Mellin transform of $x /\left(1-x+x^{2}\right)$ for $n=6 N$ is given by

$$
\begin{aligned}
\mathbf{M}\left[\frac{x}{1-x+x^{2}}\right](6 N)= & \frac{\left(4+38 N-47 N^{2}+127 N^{3}+90 N^{4}-1404 N^{5}-1080 N^{6}\right)}{12 N(2 N-1)(2 N+1)(3 N-1)(3 N+2)(6 N-5)(6 N+1)} \\
& +\frac{2 \pi}{3 \sqrt{3}(6 N-5)}+\frac{1}{6 N-5}\left[\frac{1}{3} \sum_{k=1}^{N} \frac{1}{1+2 k}+\frac{1}{2} \sum_{k=1}^{N} \frac{1}{2+3 k}\right. \\
& \left.+\sum_{k=1}^{N} \frac{1}{1+6 k}-\frac{1}{6} S_{1}(N)\right],
\end{aligned}
$$

as an example. Here cyclotomic harmonic sums such as $\sum_{k=1}^{N} \frac{1}{2+3 k}$ emerge, i.e. harmonic sums exhibiting a cyclic pattern of missing terms in their summands. New special constants are obtained from the cyclotomic harmonic sums in the limit $N \rightarrow \infty$ and the cyclotomic harmonic polylogarithms for $x \rightarrow 1$. One of these is Catalan's constant

$$
\mathrm{C}=\sum_{l=0}^{\infty} \frac{(-1)^{l}}{(2 l+1)^{2}}
$$

Cyclotomic harmonic polylogarithms contribute to the massive form factors at 3-loop order [134,239]. Real-valued cyclotomic harmonic polylogarithms can be decomposed into complexvalued generalized harmonic polylogarithms by partial fractioning. Expressions based on the latter representations are often more complicated to deal with. One example is the significantly extended ground field in case of sum-representations of their Mellin transforms, which gives preference to the real representations.

\subsection{Generalized harmonic sums and harmonic polylogarithms}

Generalized harmonic polylogarithms were introduced as iterated integrals by Kummer [240] and also studied by Poincaré [241], see also [242-244]. These functions emerged in higher loop calculations in $[48,119]$ and the properties of them have been detailed in Ref. [49]. One considers

$$
G_{b, a_{1}, \ldots, a_{m}}(x)=\int_{0}^{x} d y g_{b}(y) G_{a_{1}, \ldots, a_{m}}(y), \quad b, a_{i} \in \mathbb{C}, \quad G_{\emptyset}=1,
$$

with

$$
g_{c}(x)=\frac{1}{x-c}
$$

Any function $1 / P(x)$, with $P(x)$ a polynomial with coefficients in $\mathbb{C}$ can be decomposed into letters $g_{c}(x)$. Therefore, the framework of generalized harmonic polylogarithms is very general. The Mellin transform of (38) leads to linear combinations of the associated nested sums,

$$
S_{c, d_{1}, \ldots, d_{m}}\left(b, a_{1}, \ldots, a_{m} ; N\right)=\sum_{k=1}^{N} \frac{b^{k}}{k^{c}} S_{d_{1}, \ldots, d_{m}}\left(a_{1}, \ldots, a_{m} ; k\right), \quad c, d_{k} \in \mathbb{N} \backslash\{0\}, b, a_{i} \in \mathbb{C}, \quad S_{\emptyset}=1 .
$$


Likewise, special associated numbers are obtained in the limits $N \rightarrow \infty, x \rightarrow 1$, if the limits exist. In single scale applications in quantum field theory the letters in (38) are often rational numbers, respectively roots of unity, in the cyclotomic case, however, due to general polynomial structures also other algebraic numbers may occur. In the presence of more than one scale they are general complex numbers. Calculating the massless 3-loop Wilson coefficients for deepinelastic scattering, generalized harmonic sums appeared in intermediary steps but canceled in the finite results [43]. This applies also to the case of the $N_{F}$-terms for the 3-loop massive OMEs [46, 245]. However, in the 3-loop pure-singlet massive OME [246] generalized harmonic sums contribute. Relations for generalized harmonic polylogarithms are also implemented in the packages nestedsums [247] and Xsummer [248]. A numerical implementation for generalized harmonic polylogarithms has been given in [249].

\subsection{Binomial and inverse binomial sums and square-root valued iter- ated integrals}

At the next level of complexity, square-root valued letters of a quadratic form with integer coefficients for iterated integrals emerge. Examples are

$$
\frac{1}{(2+x) \sqrt{x-\frac{1}{4}}}, \quad \frac{1}{(1+x) \sqrt{x} \sqrt{8-x}}
$$

cf. [51]. They are related to binomial and inverse binomial structures of the following kind

$$
\left(\begin{array}{c}
2 N \\
N
\end{array}\right)=\frac{4^{N}}{\pi} \mathbf{M}\left[\frac{1}{\sqrt{x(1-x)}}\right](N), \quad \frac{1}{N\left(\begin{array}{c}
2 N \\
N
\end{array}\right)}=\frac{1}{4^{N}} \mathbf{M}\left[\frac{1}{x \sqrt{1-x}}\right](N)
$$

in Mellin space. In Ref. [51] a large number of these letters, characterized by different classes, have been studied.

In general, the Mellin transform of the iterated integrals of these square-root valued letters are related to nested sums containing (inverse) binomial weights in front of generalized harmonic sums, like

$$
\begin{aligned}
& \sum_{i=1}^{N}\left(\begin{array}{c}
2 i \\
i
\end{array}\right)(-2)^{i} \sum_{j=1}^{i} \frac{1}{j\left(\begin{array}{c}
2 j \\
j
\end{array}\right)} S_{1,2}\left(\frac{1}{2}, 1\right)(j)=\int_{0}^{1} d x \frac{(-x)^{N}-1}{x+1} \sqrt{\frac{x}{8-x}}\left[\mathrm{H}_{\mathrm{w}_{12}, 1,0}(x)-2 \mathrm{H}_{\mathrm{w}_{13}, 1,0}(x)\right. \\
& \left.-\zeta_{2}\left(\mathrm{H}_{\mathrm{w}_{12}}(x)-2 \mathrm{H}_{\mathrm{w}_{13}}(x)\right)\right]-\frac{5 \zeta_{3}}{8 \sqrt{3}} \int_{0}^{1} d x \frac{(-2 x)^{N}-1}{x+\frac{1}{2}} \sqrt{\frac{x}{4-x}}+c_{1} \int_{0}^{1} d x \frac{(-8 x)^{N}-1}{x+\frac{1}{8}} \sqrt{\frac{x}{1-x}} \\
& c_{1}=\frac{1}{\pi} \sum_{j=1}^{\infty} \frac{1}{j\left(\begin{array}{c}
2 j \\
j
\end{array}\right)} S_{1,2}\left(\frac{1}{2}, 1\right)(j) \approx 0.10184720 \ldots
\end{aligned}
$$

with $\mathrm{H}_{b, \vec{a}}(x)=\int_{x}^{1} d y f_{b}(y) \mathrm{H}_{\vec{a}}(y)$. Algorithms to compute the (inverse) Mellin transform in terms of indefinite nested sums/integrals are available in HarmonicSums [160]; note that these algorithms are based on linear recurrence and differential equation solving as described in Subsections 4.7 and 4.9. Infinite (inverse) binomial sums depending on one parameter $\xi \in \mathbb{C}$ have been 
also studied in [250,251]. Finite binomial sums appear in various massive calculations, see e.g. Refs. [142, 252,253].

In this context also new special constants are appearing, as e.g.

$$
\operatorname{arccot}(\sqrt{7}), \quad \operatorname{Li}_{n}\left(-\frac{1}{4}\right)
$$

and many others.

One may think of to rationalize the root-valued letters in trying to map the problem to Kummer-Poincaré iterated integrals. This is indeed possible for a series of cases. Concerning all the cases discussed in Ref. [51], a successful transformation could, however, not be found, cf. [254].

Finally, we would like to mention, that some elliptic solutions in $x$ space are hypergeometric in $N$-space. The Mellin transform of the following elliptic integrals of the first and the second kind obey $[61,183]$

$$
\mathbf{M}[\mathbf{K}(1-z)](N)=\frac{2^{4 N+1}}{(1+2 N)^{2}\left(\begin{array}{c}
2 N \\
N
\end{array}\right)^{2}}, \quad \mathbf{M}[\mathbf{E}(1-z)](N)=\frac{2^{4 N+2}}{(1+2 N)^{2}(3+2 N)\left(\begin{array}{c}
2 N \\
N
\end{array}\right)^{2}} .
$$

Eqs. (46) show that square-root valued iterated integrals and (inverse) binomial sum representations already have a close relation to elliptic integrals, cf. Section 7.

\subsection{Square-root valued iterated integrals: two scales}

In two-scale problems, which factorize at first order, the Feynman integrals can be represented in terms as iterated integrals of the kind

$$
G_{l, k_{1}, \ldots, k_{m}}(\eta ; x)=\int_{0}^{x} d y h_{l}(y, \eta) G_{k_{1}, \ldots, k_{m}}(\eta ; y), \quad G_{\emptyset}=1,
$$

where $l, k_{i}$ label the respective letters and $\eta$ denotes the ratio of the two scales. The functions $h_{l}(y, \eta)$ are rational or square-root valued in known examples [255-257]. The associated $N$-representations are obtained again by a Mellin transform. In some cases one finds sum representations, usually of generalized binomially weighted sums. However, there are also cases in which the $N$-space solution has no nested sum-product representation but contains higher transcendental functions in $N,[256]$. A typical iterated integral of the kind (47) is given by

$$
\begin{aligned}
& G\left[\left\{\frac{\sqrt{(1-x) x}}{1-x(1-\eta)}, \frac{1}{1-x}\right\}, z\right]=\frac{1}{(1-\eta)^{2}}\left[-i\left[\eta \operatorname{Li}_{2}\left(-(\sqrt{1-z}+i \sqrt{z})^{2}\right)\right.\right. \\
& +\sqrt{\eta} \operatorname{Li}_{2}\left(\frac{\left(1-\frac{i \sqrt{z}}{\sqrt{1-z}}\right) \sqrt{\eta}}{\sqrt{\eta}-1}\right)-\sqrt{\eta} \operatorname{Li}_{2}\left(\frac{\left(\frac{i \sqrt{z}}{\sqrt{1-z}}+1\right) \sqrt{\eta}}{\sqrt{\eta}-1}\right)-\sqrt{\eta} \operatorname{Li}_{2}\left(\frac{\left(1-\frac{i \sqrt{z}}{\sqrt{1-z}}\right) \sqrt{\eta}}{\sqrt{\eta}+1}\right) \\
& \left.+\sqrt{\eta} \operatorname{Li}_{2}\left(\frac{\left(\frac{i \sqrt{z}}{\sqrt{1-z}}+1\right) \sqrt{\eta}}{\sqrt{\eta}+1}\right)+\operatorname{Li}_{2}\left(-(\sqrt{1-z}+i \sqrt{z})^{2}\right)\right]+(\eta-1) \sqrt{(1-z) z} \\
& +i(\eta+1) \arcsin (\sqrt{z})+(1-\eta) \arcsin (\sqrt{z})+2(\eta+1) \ln (2) \arcsin (\sqrt{z})+\ln (1-z) \\
& \times\left[(1-\eta) \sqrt{(1-z) z}+2 \sqrt{\eta} \arctan \left(\frac{\sqrt{\eta z}}{\sqrt{1-z}}\right)\right]+\ln \left(\frac{1-\sqrt{\eta}}{\sqrt{\eta}+1}\right)\left(\pi \sqrt{\eta}-\frac{1}{2} i(\eta+1) \zeta_{2}\right.
\end{aligned}
$$




$$
\left.\left.-2 \sqrt{\eta} \arctan \left(\frac{\sqrt{1-z}}{\sqrt{z}}\right)\right)\right]
$$

with the letters $\sqrt{(1-x) x} /(1-x(1-\eta))$ and $1 /(1-x)$, cf. [257]. The associated constants are functions of $\eta$. Furthermore, also usual harmonic polylogarithms at new algebraic arguments contribute, cf. [257].

\section{$7 \quad$ Non First Order Factorizing Systems}

At higher and higher orders in perturbation theory, at least in the massive case, the system of differential equations for the master integrals will not exhibit first order factorizations at a certain level of complexity. In various cases this will be not the case for the associated system of difference equations, through a Mellin transform, either. There are a series of well-known examples in the literature as the sun-rise integral, cf. e.g. [53,55-59], the kite-integral [60,258], the 3-loop QCDcorrections to the $\rho$-parameter [61], and the 3-loop QCD corrections to the massive operator matrix element $A_{Q g}$ [135], to which non first order factorizing systems contribute.

In decoupling the corresponding system of differential equations [93,158] one obtains 2nd order factors of the associated scalar equations, which cannot be reduced further. More precisely, this associated scalar differential equation of order two contains more than three singularities. It is formally a Heun differential equation [259]. One can write the corresponding solution also using ${ }_{2} F_{1}$-functions with rational argument $[61,260]$ and rational parameters. It is now interesting to see whether these solutions can be expressed in terms of complete elliptic integrals, which can be checked algorithmically using the triangle group [261]. In the examples mentioned before one can find representations in terms of complete elliptic integrals of the first and second kind, $\mathbf{K}$ and $\mathbf{E}$, cf. [52,138]. A related question is, whether an argument translation allows for a representation through only $\mathbf{K}$. This is possible under certain conditions, cf. [262,263]. In the case of the 3-loop QCD-corrections to the $\rho$-parameter, however, this is not possible.

A homogeneous ${ }_{2} F_{1}$-solution $\psi_{k}^{(0)}(x), k=1,2$ of the 2 nd order differential equation is represented by the integral (6), which cannot be rewritten such, that it depends on $x$ just through the integral boundaries. This integral is therefore non-iterative w.r.t. $x$. The inhomogeneous solution reads

$$
\psi(x)=\psi_{1}^{(0)}(x)\left[C_{1}-\int d x \psi_{2}^{(0)}(x) \frac{N(x)}{W(x)}\right]+\psi_{2}^{(0)}(x)\left[C_{2}+\int d x \psi_{1}^{(0)}(x) \frac{N(x)}{W(x)}\right],
$$

with $N(x)$ and $W(x)$ the inhomogeneity and the Wronskian and $C_{1,2}$ are the integration constants. Through partial integration the ratio $N(x) / W(x)$ can be transformed into an iterative integral. Since $\psi_{k}^{(0)}(x)$ cannot be written as iterative integrals, $\psi(x)$ is obtained as an iterative non-iterative integral [61,264] of the type

$$
\begin{aligned}
\mathbb{H}_{a_{1}, \ldots, a_{m-1} ; a_{m}, F_{m}\left(r\left(y_{m}\right)\right), a_{m+1}, \ldots a_{q}}(x)= & \int_{0}^{x} d y_{1} f_{a_{1}}\left(y_{1}\right) \int_{0}^{y_{1}} d y_{2} \ldots \int_{0}^{y_{m-1}} d y_{m} f_{a_{m}}\left(y_{m}\right) F_{m}\left[r\left(y_{m}\right)\right] \\
& \times H_{a_{m+1}, \ldots, a_{q}}\left(y_{m}\right),
\end{aligned}
$$

with $r(x)$ a rational function and $F_{m}$ a non-iterative integral. Notice, that more than one non-iterative integral can contribute and $F_{m}$ denotes any non-iterative integral, implying a very general representation, cf. [61]. ${ }^{8}$ In Ref. [266] an $\varepsilon$-form for the Feynman diagrams of elliptic cases

${ }^{8}$ This representation has been used in a more special form also in [265] later. 
has been found recently. However, transcendental letters contribute here. This is in accordance with our earlier finding, Eq. (50), which, as well is an iterative integral over all objects between the individual iterations and to which now also the non-iterative higher transcendental functions $F_{m}\left[r\left(y_{m}\right)\right]$ contribute.

One may obtain fast convergent representations of $\mathbb{H}(x)$ by overlapping series expansions around $x=x_{0}$ outside possible singularities, see Ref. [61] for details.

Because in many cases elliptic solutions are obtained one may transform the kinematic variable $x$ occurring as $\mathbf{K}\left(k^{2}\right)=\mathbf{K}(r(x))$ into the variable $q=\exp [i \pi \tau]$ analytically with

$$
k^{2}=r(x)=\frac{\vartheta_{2}^{4}(q)}{\vartheta_{3}^{4}(q)},
$$

by applying a higher order Legendre-Jacobi transformation. Here $\vartheta_{l}, l=1, \ldots, 4$ denote Jacobi's $\vartheta$-functions and $\operatorname{Im}(\tau)>0$. In this way Eqs. (49) and (50) are rewritten in terms of the new variable. The integrands are given by products of meromorphic modular forms, cf. [62-64], which are given by linear combinations of ratios of Dedekind's $\eta$-function

$$
\eta(\tau)=q^{\frac{1}{12}} \prod_{k=1}^{\infty}\left(1-q^{2 k}\right) .
$$

Depending on the largest multiplier $k \in \mathbb{N}, k_{m}$, of $\tau$ in the argument of the $\eta$-function, the solution transforms under the congruence subgroup $\Gamma_{0}\left(k_{m}\right)$. One can perform Fourier expansions in $q$ around the different cusps of the problem, cf. [267,268]. This representation is a uniform one w.r.t. the singularities if compared to the former ${ }_{2} F_{1}$-representation. In the latter only three singularities of the problem are encoded in the ${ }_{2} F_{1}$-function, while all others are implied by the argument $r(z)$ in an asymmetric way.

In the case that the occurring modular forms are holomorphic, one obtains representations in Eisenstein series with character, while in the meromorphic case additional $\eta$-factors in the denominators are present. In the former case the $q$-integrands can be written in terms of elliptic polylogarithms in the representation

$$
\operatorname{ELi}_{n, m}(x, y)=\sum_{k=1}^{\infty} \sum_{l=1}^{\infty} \frac{x^{k}}{k^{n}} \frac{y^{l}}{l^{m}} q^{k l}
$$

and products thereof, cf. [58]. The corresponding $q$-integrals can be directly performed. The solution (49) usually appears for single master integrals. Other master integrals are obtained integrating further other letters, so that finally representations by $\mathbb{H}(x)$ occur. Iterated modular forms, resp. Eisenstein series, have been also discussed recently in [269, 270].

For systems of differential equations which factorize to 3rd or higher order much less is know. Formally, the representation Eq. (50) also applies there. However, one will be also interested in the more specific properties of these systems having finally concrete cases at hand.

\section{Outlook}

Various powerful computation methods exist to solve massless and massive problems in quantum field theory to 4- and 5-loop order analytically for a low number of external legs and scales involved. The mathematical understanding of the associated solution spaces made substantial progress during the last two decades and the computer-algebraic methods gained orders of magnitude during a similar period. One now has to see which structures have to be revealed next, going to even higher orders and/or allowing for more scales. 
Despite the fact that a systematic classification for sets of Feynman integrals can be obtained through their degree of decoupling of the associated systems of differential equations, cf. Section 2, just the use of differential equations makes it difficult to obtain closed form analytic solutions. This is due to the fact, that this approach is a rather inclusive one, as also others. Already in early studies of elliptic solutions, see e.g. [54], dispersive representations have been used. As well-known, cuts allow access to the specific integrand structures and, e.g., the elliptic solutions for the respective integrals were easily obtained. ${ }^{9}$ The cut-representation is equivalent to a Hilbert-transform [272-274]

$$
F(s)=\int_{-\infty}^{+\infty} \frac{d t}{t-s} f(t)
$$

splitting off one corresponding integral. In more involved cases, one might want to apply even multiple cuts to reveal the corresponding class of integrals. In this way, the dispersive approach, [275], will form an important asset also to many key problems to be dealt with in the future. In the practical calculation of Feynman diagrams, the general Hopf-algebra structure [218-221] implied by the renormalization of the corresponding quantum field theory may play a role. In general, however, all information is encoded in the specific multi-variate hyper-exponential structure of the individual integrand of the graph itself, which decides on its mathematical result and the wider class of mathematical structures to which it will finally belong. Experience shows that it is often rather difficult to determine the latter a priori, since these specific structures only unfold in course of the different integrations in most of the interesting cases.

One can imagine that more involved Feynman diagrams could lead to Abel-integrals [276]. Also integrals related to K3-surfaces [277] are expected. The development in the field of zeroand single-scale quantities during the last 25 years let us expect a rich host of mathematical structures to be unrevealed and techniques to be discovered and designed going to even higher loop orders and allowing to study also cases in which a few more invariants are present during the decades to come.

The theoretical predictions for the measurements planned at the FCC have to have an accuracy below the experimental errors which can be achieved. This will require at least one order of magnitude better complete calculations in the respective quantum field theory, and in some important cases even more. To achieve this will require important improvements of what is technically possible at present, also in case of analytic calculations. The present experience has to be maintained for the future and to be significantly extended. This will also require an even closer cooperation between physicists and mathematicians and will include the solution of unprecedented tasks within computer algebra as well. As experience tells, these enormous tasks are by far not self-organizing and are of very long term nature to be taken into account in the thorough planning of this collider project. Large-scale and long-term research of this kind is therefore often performed with a strong involvement of leading international research centers. A series of important calculations for HERA and the LHC spanned over two decades, which is setting a lower bound to what will be needed at the FCC. A substantial theoretical community will be needed to solve these tasks. In observing the fantastic, strongly interdisciplinary leaps forward having been made since the late 1980ies, it seems to be possible, but a great effort is needed to master this adventure. The theoretical and mathematical insight which will be gained will be very large. The task is indispensable, however, to be able to interprete the experimental precision measurements at a facility like the FCC.

Acknowledgment. We would like to thank J. Ablinger, D.B. Broadhurst, D. Kreimer, and

\footnotetext{
${ }^{9}$ For the well studied simpler one-loop case, see e.g. [271].
} 
P. Marquard for discussions. This work was supported in part by the Austrian Science Fund (FWF) grant SFB F50 (F5009-N15).

\section{References}

[1] The Future Circular Collider, https://en.wikipedia.org/wikiFuture_Circular_Collider; TH FCC-ee design study, http://tlep.web.cern.ch.

[2] G. 't Hooft and M. J. G. Veltman, Nucl. Phys. B153 (1979) 365-401.

[3] G. 't Hooft and M. J. G. Veltman, NATO Sci. Ser. B 4 (1974) 177-322.

[4] M.J.G. Veltman, Diagrammatica: The Path to Feynman rules, Cambridge Lect. Notes Phys. 4, (Cambridge University Press, Cambridge, 1994).

[5] A. Denner and S. Dittmaier, Nucl. Phys. B658 (2003) 175-202, [hep-ph/0212259].

[6] T. Binoth, J. P. Guillet, G. Heinrich, E. Pilon, and T. Reiter, Comput. Phys. Commun. 180 (2009) 2317-2330, [arXiv:0810.0992[hep-ph]].

[7] J. Fleischer and T. Riemann, Phys. Rev. D83 (2011) 073004, [arXiv:1009.4436[hep-ph]].

[8] J. Fleischer and T. Riemann, Phys. Lett. B707 (2012) 375-380, [arXiv:1111.5821[hep-ph]].

[9] J. Fleischer, F. Jegerlehner, and O. V. Tarasov, Nucl. Phys. B672 (2003) 303-328, [hep-ph/0307113].

[10] J. Blümlein, K. H. Phan, and T. Riemann, Acta Phys. Pol. B48 (2017) 2313-2320, [arXiv:1711.05510[hep$\mathrm{ph}]]$.

[11] J. Blümlein, K. H. Phan, and T. Riemann, DESY 17-079.

[12] F. Klein, Vorlesungen über die hypergeometrische Funktion, Wintersemester 1893/94, Die Grundlehren der Mathematischen Wissenschaften 39, (Springer, Berlin, 1933).

[13] W.N. Bailey, Generalized Hypergeometric Series, (Cambridge University Press, Cambridge, 1935).

[14] L.J. Slater, Generalized hypergeometric functions, (Cambridge University Press, Cambridge, 1966).

[15] P. Appell and J. Kampé de Fériet, Fonctions Hypergéométriques et Hypersphériques, Polynomes D' Hermite, (Gauthier-Villars, Paris, 1926).

[16] P. Appell, Les Fonctions Hypergëométriques de Plusieur Variables, (Gauthier-Villars, Paris, 1925).

[17] J. Kampé de Fériet, La fonction hypergëométrique, (Gauthier-Villars, Paris, 1937).

[18] H. Exton, Multiple Hypergeometric Functions and Applications, (Ellis Horwood, Chichester, 1976).

[19] H. Exton, Handbook of Hypergeometric Integrals, (Ellis Horwood, Chichester, 1978).

[20] M.J. Schlosser, in: Computer Algebra in Quantum Field Theory: Integration, Summation and Special Functions, C. Schneider, J. Blümlein, Eds., p. 305-324, (Springer, Wien, 2013) [arXiv:1305.1966 [math.CA]].

[21] H.M. Srivastava and P.W. Karlsson, Multiple Gaussian Hypergeometric Series, (Ellis Horwood, Chicester, 1985).

[22] G. Lauricella, Rediconti del Circolo Matematico di Palermo, 7 (S1) (1893) 111-158.

[23] S. Saran, Ganita 5 (1954) 77-91.

[24] S. Saran, Acta Math. 93 (1955) 293-312.

[25] J. Blümlein, PoS (RADCOR2011) 048, [arXiv:1205.4991[hep-ph]].

[26] F. A. Berends, W. L. van Neerven, and G. J. H. Burgers, Nucl. Phys. B297 (1988) 429-478, [Erratum: Nucl. Phys. B304 (1988) 921-922].

[27] J. Blümlein, A. De Freitas, and W. van Neerven, Nucl. Phys. B855 (2012) 508-569, [arXiv:1107.4638[hep$\mathrm{ph}]]$.

[28] R. Hamberg, W. L. van Neerven, and T. Matsuura, Nucl. Phys. B359 (1991) 343-405, [Erratum: Nucl. Phys. B644 (2002) 403-404]. 
[29] E. B. Zijlstra and W. L. van Neerven, Nucl. Phys. B383 (1992) 525-574.

[30] M. Buza, Y. Matiounine, J. Smith, R. Migneron, and W. L. van Neerven, Nucl. Phys. B472 (1996) 611-658, [hep-ph/9601302].

[31] L. Lewin, Dilogarithms and associated functions, (Macdonald, London, 1958).

[32] L. Lewin, Polylogarithms and associated functions, (North Holland, New York, 1981).

[33] N. Nielsen Nova Acta Leopold. XC (1909) Nr. 3, 125-211.

[34] K.S. Kölbig, J.A. Mignoco, and E. Remiddi, BIT 10 (1970) 38-74.

[35] K. S. Kölbig, SIAM J. Math. Anal. 17 (1986) 1232-1258.

[36] A. Devoto and D. W. Duke, Riv. Nuovo Cim. 7N6 (1984) 1-39.

[37] R. Harlander and M. Steinhauser, Prog. Part. Nucl. Phys. 43 (1999) 167-228, hep-ph/9812357.

[38] J. Vermaseren, Int. J. Mod. Phys. A14 (1999) 2037-2076, [hep-ph/9806280].

[39] J. Blümlein and S. Kurth, Phys. Rev. D60 (1999) 014018, [hep-ph/9810241].

[40] E. Remiddi and J. Vermaseren, Int. J. Mod. Phys. A15 (2000) 725-754, [hep-ph/9905237].

[41] S. Moch, J. Vermaseren, and A. Vogt, Nucl. Phys. B688 (2004) 101-134, [hep-ph/0403192].

[42] A. Vogt, S. Moch, and J. Vermaseren, Nucl. Phys. B691 (2004) 129-181, [hep-ph/0404111].

[43] J. Vermaseren, A. Vogt, and S. Moch, Nucl. Phys. B724 (2005) 3-182, [hep-ph/0504242].

[44] J. Ablinger, A. Behring, J. Blümlein, A. De Freitas, A. von Manteuffel, and C. Schneider, Nucl. Phys. B922 (2017) 1-40, [arxiv:1705.01508[hep-ph]].

[45] I. Bierenbaum, J. Blümlein, S. Klein, and C. Schneider, Nucl. Phys. B803 (2008) 1-41, [hep-ph/0803.0273].

[46] J. Ablinger, J. Blümlein, S. Klein, C. Schneider, and F. Wißbrock, Nucl. Phys. B844 (2011) 26-54, [hep-ph/1008.3347].

[47] J. Ablinger, A. Behring, J. Blümlein, A. De Freitas, A. Hasselhuhn, A. von Manteuffel, M. Round, C. Schneider, and F. Wißbrock, Nucl. Phys. B886 (2014) 733-823, [arXiv:1406.4654[hep-ph]].

[48] S. Moch, P. Uwer, and S. Weinzierl, J. Math. Phys. 43 (2002) 3363-3386, [hep-ph/0110083].

[49] J. Ablinger, J. Blümlein, and C. Schneider, J. Math. Phys. 54 (2013) 082301, [arXiv: 1302.0378 [math-ph]].

[50] J. Ablinger, J. Blümlein, and C. Schneider, J. Math. Phys. 52 (2011) 102301, [arXiv: 1105.6063 [math-ph]].

[51] J. Ablinger, J. Blümlein, C. G. Raab, and C. Schneider, J. Math. Phys. 55 (2014) 112301, [arXiv: 1407.1822 [hep-th]].

[52] F.G. Tricomi, Elliptische Funktionen, (Geest \& Portig, Leipzig, 1948); übersetzt und bearbeitet von M. Krafft.

[53] D. J. Broadhurst, J. Fleischer, and O. V. Tarasov, Z. Phys. C60 (1993) 287-302, [hep-ph/9304303].

[54] S. Laporta and E. Remiddi, Nucl. Phys. B704 (2005) 349-386, [hep-ph/0406160].

[55] S. Bloch and P. Vanhove, J. Number Theor. 148 (2015) 328-364, [hep-th/1309.5865].

[56] L. Adams, C. Bogner, and S. Weinzierl, J. Math. Phys. 54 (2013) 052303, [hep-ph/1302.7004].

[57] L. Adams, C. Bogner, and S. Weinzierl, J. Math. Phys. 55 (2014), no. 10 102301, [hep-ph/1405.5640].

[58] L. Adams, C. Bogner, and S. Weinzierl, J. Math. Phys. 56 (2015), no. 7 072303, [hep-ph/1504.03255].

[59] L. Adams, C. Bogner, and S. Weinzierl, J. Math. Phys. 57 (2016), no. 3 032304, [hep-ph/1512.05630].

[60] L. Adams, C. Bogner, A. Schweitzer, and S. Weinzierl, J. Math. Phys. 57 (2016), no. 12122302 , [hep$\mathrm{ph} / 1607.01571]$.

[61] J. Ablinger, J. Blümlein, A. De Freitas, M. van Hoeij, E. Imamoglu, C.G. Raab, C.S. Radu, and C. Schneider, Iterated Elliptic and Hypergeometric Integrals for Feynman Diagrams, J. Math. Phys. (2018) in print, [arXiv:1706.01299[hep-th]].

[62] J.-P. Serre, A Course in Arithmetic, (Springer, Berlin, 1973). 
[63] H. Cohen and F. Strömberg, Modular Forms, A Classical Approach, Graduate Studies in Mathematics 179 (AMS, providence, RI, 2017).

[64] K. Ono, The Web of Modularity: Arithmetic of the Coefficients of Modular Forms and $q$-series, CBMS Regional Conference Series in Mathematics, 102 (AMS, Providence, RI, 2004).

[65] E.W. Barnes, Quarterly Journal of Mathematics 41 (1910) 136-140.

[66] H. Mellin, Math. Ann. 68, no. 3 (1910) 305-337.

[67] M. Czakon, Comput. Phys. Commun. 175 (2006) 559-571, [hep-ph/0511200].

[68] A. Smirnov and V. Smirnov, Eur. Phys. J. C62 (2009) 445-449, [arXiv: 0901.0386 [hep-ph]].

[69] J. Gluza, K. Kajda, and T. Riemann, Comput. Phys. Commun. 177 (2007) 879-893, [arXiv:0704.2423[hep$\mathrm{ph}]$.

[70] J. Blümlein, S. Klein, C. Schneider, and F. Stan, J. Symb. Computation 47 (2012) 1267-1289, [arXiv: 1011.2656 [cs.SC]].

[71] M. Karr, J. ACM 28 (1981) 305-350.

[72] M. Bronstein, J. Symbolic Comput. 29 (2000), no. 6 841-877.

[73] C. Schneider, Symbolic Summation in Difference Fields, Ph.D. Thesis RISC, Johannes Kepler University, Linz technical report 01-17 (2001).

[74] C. Schneider, An. Univ. Timisoara Ser. Mat.-Inform. 42 (2004) 163-179.

[75] C. Schneider, J. Differ. Equations Appl. 11 (2005) 799-821.

[76] C. Schneider, Appl. Algebra Engrg. Comm. Comput. 16 (2005) 1-32.

[77] C. Schneider, J. Algebra Appl. 6 (2007) 415-441.

[78] C. Schneider, Motives, Quantum Field Theory, and Pseudodifferential Operators, Clay Mathematics Proceedings Vol. 12, eds. A. Carey, D. Ellwood, S. Paycha and S. Rosenberg, (Amer. Math. Soc) (2010), 285-308, [arXiv:0904.2323].

[79] C. Schneider, Ann. Comb. 14 (2010) 533-552, [arXiv:0808.2596].

[80] C. Schneider, in: Computer Algebra and Polynomials, Applications of Algebra and Number Theory, J. Gutierrez, J. Schicho, M. Weimann (ed.), Lecture Notes in Computer Science (LNCS) 8942 (2015), 157-191, [arXiv:1307.7887 [cs.SC]].

[81] C. Schneider, J. Symbolic Comput. 43 (2008) 611-644 [arXiv:0808.2543].

[82] C. Schneider, J. Symb. Comput. 72 (2016) 82-127, [arXiv:1408.2776 [cs.SC]].

[83] C. Schneider, J. Symb. Comput. 80 (2017) 616-664. [arXiv:1603.04285 [cs.SC]].

[84] C. Schneider, Sém. Lothar. Combin. 56 (2007) 1-36, article B56b.

[85] C. Schneider, in: Computer Algebra in Quantum Field Theory: Integration, Summation and Special Functions, Texts and Monographs in Symbolic Computation eds. C. Schneider and J. Blümlein (Springer, Wien, 2013), 325-360 [arXiv:1304.4134 [cs.SC]].

[86] K. G. Chetyrkin and F. V. Tkachov, Nucl. Phys. B192 (1981) 159-204.

[87] S. Laporta, Int. J. Mod. Phys. A15 (2000) 5087-5159, [hep-ph/0102033].

[88] C. Studerus, Comput. Phys. Commun. 181 (2010) 1293-1300, [arXiv: 0912.2546 [physics.comp-ph]].

[89] A. von Manteuffel, C. Studerus, Reduze 2 - Distributed Feynman Integral Reduction, arXiv: 1201.4330 [hep-ph].

[90] P. Marquard and D. Seidel, The Crusher algorithm, (unpublished).

[91] S. Gerhold, Uncoupling systems of linear Ore operator equations, Master's thesis, RISC, J. Kepler University, Linz, 2002.

[92] A. Bostan, F. Chyzak, É. de Panafieu, Complexity Estimates for Two Uncoupling Algorithms, Proceedings of ISSAC'13, Boston, June 2013. 
[93] B. Zürcher, Rationale Normalformen von pseudo-linearen Abbildungen, Master's thesis, Mathematik, ETH Zürich (1994).

[94] M. Czakon and A. Mitov, Nucl. Phys. B824 (2010) 111-135, [arXiv:0811.4119[hel-ph].

[95] P. Nogueira, J. Comput. Phys. 105 (1993) 279-289.

[96] T. van Ritbergen, A. Schellekens, and J. Vermaseren, Int. J. Mod. Phys. A14 (1999) 41-96, [hep$\mathrm{ph} / 9802376]$.

[97] N. Nakanishi, Graph Theory and Feynman Integrals, (Gordon and Breach, New York, 1971).

[98] S. Lefschetz, Applications of Algebraic Topology: Graphs and Networks, the Picard-Lefschetz Theory an Feynman Integrals, (Springer, Berlin, 1975).

[99] C. Bogner and S. Weinzierl, Int. J. Mod. Phys. A25 (2010) 2585-2618, [arXiv:1002.3458[hep-ph]].

[100] J.A.M. Vermaseren, New features of FORM, math-ph/0010025.

[101] M. Tentyukov, D. Fliegner, M. Frank, A. Onischenko, A. Retey, H.M. Staudenmaier, and J.A.M. Vermaseren, ParFORM: Parallel version of the symbolic manipulation program FORM, cs/0407066.

[102] M. Tentyukov and J. Vermaseren, Comput. Phys. Commun. 181 (2010) 1419-1427, [hep-ph/0702279].

[103] B. Ruijl, U. Takahiro, and J.A.M. Vermaseren, FORM version 4.2, arXiv: 1707.06453[hep-ph].

[104] C. Anastasiou and A. Lazopoulos, JHEP 07 (2004) 046, [hep-ph/0404258].

[105] A. Smirnov, JHEP 10 (2008) 107, [arXiv: 0807.3243[hep-ph]].

[106] K. Hepp, Commun. Math. Phys. 2 (1966) 301-326.

[107] T. Binoth and G. Heinrich, Nucl. Phys. B585 (2000) 741-759, [hep-ph/0004013].

[108] Z. Nagy and D. E. Soper, Phys. Rev. D74 (2006) 093006, [hep-ph/0610028].

[109] C. Anastasiou, S. Beerli, and A. Daleo, JHEP 05 (2007) 071, [hep-ph/0703282].

[110] A. V. Smirnov and M. N. Tentyukov, Comput. Phys. Commun. 180 (2009) 735-746, [arXiv: 0807.4129[hep$\mathrm{ph}]]$.

[111] J. Carter and G. Heinrich, Comput. Phys. Commun. 182 (2011) 1566-1581, [arXiv:1011.5493[hep-ph]].

[112] A. V. Smirnov, V. A. Smirnov, and M. Tentyukov, Comput. Phys. Commun. 182 (2011) 790-803, [arXiv:0912.0158[hep-ph]].

[113] S. Becker, C. Reuschle, and S. Weinzierl, JHEP 12 (2010) 013, [arXiv:1010.4187[hep-ph]].

[114] S. Becker, C. Reuschle, and S. Weinzierl, JHEP 1207 (2012) 090, [arXiv:1205.2096[hep-ph]].

[115] S. Becker, D. Götz, C. Reuschle, C. Schwan, and S. Weinzierl, Phys. Rev. Lett. 108 (2012) 032005, [arXiv:1111.1733[hel-ph]].

[116] A. V. Smirnov, Comput. Phys. Commun. 204 (2016) 189-199, [arXiv:1511.03614[hep-ph]].

[117] S. Borowka, T. Gehrmann, and D. Hulme, arXiv:1804.06824[hep-ph].

[118] H.R.P. Ferguson and D.H. Bailey, A Polynomial Time, Numerically Stable Integer Relation Algorithm, RNR Techn. Rept. RNR-91-032, Jul. 14, 1992.

[119] J. M. Borwein, D. M. Bradley, D. J. Broadhurst, and P. Lisonek, Trans. Am. Math. Soc. 353 (2001) 907-941, [math/9910045].

[120] S. Laporta, Phys. Lett. B772 (2017) 232-238, [arXiv:1704.06996[hep-ph]].

[121] T. Luthe, A. Maier, P. Marquard, and Y. Schröder, JHEP 10 (2017) 166, [arXiv: 1709.07718 [hep-ph]].

[122] J. Blümlein, D. Broadhurst, and J. Vermaseren, Comput. Phys. Commun. 181 (2010) 582-625, [arXiv: 0907.2557 [math-ph]].

[123] P. A. Baikov, K. G. Chetyrkin, and J. H. Kühn, Phys. Rev. Lett. 118 (2017), no. 8 082002, [arXiv: 1606.08659 [hep-ph]]. 
[124] F. Herzog, B. Ruijl, T. Ueda, J. Vermaseren, and A. Vogt, JHEP 02 (2017) 090, [arXiv: 1701.01404 [hep-ph]].

[125] I. Bierenbaum, J. Blümlein, and S. Klein, Nucl. Phys. B820 (2009) 417-482, [arXiv:0904.3563[hep-ph]].

[126] N.E. Nörlund, Vorlesungen über Differenzenrechnung, (Springer, Berlin, 1924); reprinted by (Chelsea Publishing Company, New York, 1954).

[127] S. A. Larin, T. van Ritbergen, and J. A. M. Vermaseren, Nucl. Phys. B427 (1994) 41-52.

[128] S. A. Larin, P. Nogueira, T. van Ritbergen, and J. A. M. Vermaseren, Nucl. Phys. B492 (1997) 338-378, [hep-ph/9605317].

[129] A. Retey and J. A. M. Vermaseren, Nucl. Phys. B604 (2001) 281-311, [hep-ph/0007294].

[130] J. Blumlein and J. A. M. Vermaseren, Phys. Lett. B606 (2005) 130-138, [hep-ph/0411111].

[131] M. Kauers, M. Jaroschek, and F. Johansson, in: Computer Algebra and Polynomials, Editors: J. Gutierrez, J. Schicho, Josef, M. Weimann, Lecture Notes in Computer Science 8942 (Springer, Berlin, 2015) 105-125, [arXiv:1306.4263 [cs.SC]].

[132] Sage, http://www.sagemath.org/.

[133] J. Blümlein, M. Kauers, S. Klein, and C. Schneider, Comput. Phys. Commun. 180 (2009) 2143-2165, [arXiv:0902.4091[hep-ph]].

[134] J. Ablinger, J. Blümlein, P. Marquard, N. Rana, and C. Schneider, DESY 18-052, DESY 18-053.

[135] J. Ablinger, A. Behring, J. Blümlein, A. De Freitas, A. von Manteuffel, and C. Schneider, PoS (QCDEV2017) 031 [arXiv:1711.07957[hep-ph]].

[136] R. Hamberg, Second order gluonic contributions to physical quantities, Ph.D. Thesis, Leiden University, 1991.

[137] I. Bierenbaum, J. Blümlein, and S. Klein, Nucl. Phys. B780 (2007) 40-75, [hep-ph/0703285].

[138] E.T. Whittaker and G.N. Watson, A Course of Modern Analysis, (Cambridge University Press, Cambridge, 1996), reprint of 4th edition (1927).

[139] C. Anastasiou, E. W. N. Glover, and C. Oleari, Nucl. Phys. B572 (2000) 307-360, [hep-ph/9907494].

[140] C. Anastasiou, E. W. N. Glover, and C. Oleari, Nucl. Phys. B565 (2000) 445-467, [hep-ph/9907523].

[141] J. Ablinger, J. Blümlein, A. Hasselhuhn, S. Klein, C. Schneider, and F. Wißbrock, Nucl. Phys. B864 (2012) 52-84, [arXiv:1206.2252[hep-ph]].

[142] J. Ablinger, A. Behring, J. Blümlein, A. De Freitas, A. von Manteuffel, and C. Schneider, Comput. Phys. Commun. 202 (2016) 33-112, [arXiv:1509.08324[hep-ph]].

[143] J. Gluza, K. Kajda, T. Riemann, and V. Yundin, Eur. Phys. J. C71 (2011) 1516, [arXiv:1010.1667[hep-ph]].

[144] I. Dubovyk, J. Gluza, T. Riemann, and J. Usovitsch, PoS (LL2016) 034 [arXiv:1607.07538[hep-ph].

[145] I. Dubovyk, A. Freitas, J. Gluza, T. Riemann, and J. Usovitsch, Phys. Lett. B762 (2016) 184-189, [arXiv:1607.08375[hep-ph]].

[146] A. von Manteuffel, E. Panzer, and R. M. Schabinger, JHEP 02 (2015) 120, [arXiv:1411.7392[hep-ph]].

[147] F. Brown, Commun. Math. Phys. 287 (2009) 925-958, [arXiv:0804.1660[math.AG]].

[148] E. Panzer, Comput. Phys. Commun. 188 (2015) 148-166, [arXiv:1403.3385[hep-th]].

[149] J. Ablinger, J. Blümlein, C. Raab, C. Schneider, and F. Wißbrock, Nucl. Phys. B885 (2014) 409-447, [arXiv:1403.1137[hep-ph]].

[150] F.Ph. Wißbrock, $O\left(\alpha_{s}^{3}\right)$ contributions to the heavy flavor Wilson coefficients of the structure function $F_{2}\left(x, Q^{2}\right)$ at $Q^{2} \gg m^{2}$, Ph.D. Thesis, TU Dortmund, 2015.

[151] G. Almkvist and D. Zeilberger, J. Symb. Comp. 10 (1990) 571-591.

[152] M. Apagodu and D. Zeilberger, Adv. Appl. Math. (Special Regev Issue), 37 (2006) 139-152. 
[153] J. Ablinger, Computer Algebra Algorithms for Special Functions in Particle Physics, Ph.D. Thesis, Linz U. (2012) arXiv:1305.0687[math-ph].

[154] A. V. Kotikov, Phys. Lett. B254 (1991) 158-164.

[155] Z. Bern, L. J. Dixon, and D. A. Kosower, Phys. Lett. B302 (1993) 299-308, [Erratum: Phys. Lett. B318, (1993) 649], [hep-ph/9212308].

[156] E. Remiddi, Nuovo Cim. A110 (1997) 1435-1452, [hep-th/9711188].

[157] T. Gehrmann and E. Remiddi, Nucl. Phys. B580 (2000) 485-518, [hep-ph/9912329].

[158] J. Ablinger and J. Blümlein, in: Computer Algebra in Quantum Field Theory. Integration, Summation and Special Functions, (Springer, Wien, 2012), 1-32, Eds. C. Schneider and J. Blümlein, [arXiv: 1304.7071 [math-ph]].

[159] S. A. Abramov and M. Petkovšek, D'Alembertian solutions of linear differential and difference equations, Proc. ISSAC'94, 169-174, ed. J. von zur Gathen, (ACM Press, 1994).

[160] J. Ablinger, PoS (RADCOR2017) 001 [arXiv:1801.01039 [cs.SC]].

[161] M.F. Singer, American Journal of Mathematics, 103 (4) (1981) 661-682.

[162] J.J. Kovacic, J. Symb. Computation 2 (1986) 3-43.

[163] J. Ablinger, A. Behring, J. Blümlein, G. Falcioni, A. De Freitas, P. Marquard, N. Rana, and C. Schneider, Phys. Rev. D (in print) (2018) [arxiv:1712.09889[hep-ph]].

[164] C.G. Raab, Definite Integration in Differential Fields, PhD Thesis, Linz U., 2012.

[165] A.V. Kotikov, The Property of maximal transcendentality in the N=4 Supersymmetric Yang-Mills, In Subtleties in quantum field theory, ed. D. Diakonov, p. 150-174, [arXiv:1005.5029 [hep-th]].

[166] J. M. Henn, Phys. Rev. Lett. 110 (2013) 251601, [arXiv:1304.1806[hep-th]].

[167] J. M. Henn, J. Phys. A48 (2015) 153001, [arXiv:1412.2296[hep-ph]].

[168] В.Е. Захаров, С.В. Манаков, С.П. Новиков, Л.П. Питаевский, Теориа Солитонов: метод обратной задацчи, (Наука, Москва, 1980).

[169] S.Yu. Sakovich, J. Phys. A: Math. Gen. 28 (1995) 2861-2869.

[170] S. Lie, Vorlesungen über Differentialgleichungen mit bekannten infinitesimalen Transformationen, (Teubner, Leipzig, 1891).

[171] R. N. Lee, JHEP 04 (2015) 108, [arXiv:1411.0911[hep-ph]].

[172] M. Prausa, Comput. Phys. Commun. 219 (2017) 361-376, [arXiv:1701.00725[hep-ph]].

[173] O. Gituliar and V. Magerya, Comput. Phys. Commun. 219 (2017) 329-338, [arXiv:1701.04269[hep-ph]].

[174] C. Meyer, Comput. Phys. Commun. 222 (2018) 295-312, [arXiv:1705.06252[hep-ph]].

[175] S.G. Gorishnii, S.A. Larin, L.R. Surguladze, F.V. Tkachov, Comput. Phys. Commun. 55 (1989) 381-408; S.A. Larin, F.V. Tkachov and .A.M. Vermaseren, The FORM version of MINCER, NIKHEF-H-91-18.

[176] P. A. Baikov, Phys. Lett. B385 (1996) 404-410, [hep-ph/9603267].

[177] V. A. Smirnov and M. Steinhauser, Nucl. Phys. B672 (2003) 199-221, [hep-ph/0307088].

[178] B. Ruijl, T. Ueda, and J. A. M. Vermaseren, [arXiv:1704.06650[hep-ph]].

[179] F. Herzog and B. Ruijl, JHEP 05 (2017) 037, [arXiv:1703.03776[hep-th]].

[180] R. Harlander, T. Seidensticker, and M. Steinhauser, Phys. Lett. B426 (1998) 125-132, [hep-ph/9712228].

[181] T. Seidensticker, Automatic application of successive asymptotic expansions of Feynman diagrams, in: Proc. 6th International Workshop on New Computing Techniques in Physics Research, Crete, Greece, April 1999, hep-ph/9905298.

[182] J. Blümlein and C. Schneider, Phys. Lett. B771 (2017) 31-36, [arXiv:1701.04614[hep-ph]].

[183] J. Ablinger, J. Blümlein, and C. Schneider, J. Phys. Conf. Ser. 523 (2014) 012060, [arXiv: 1310.5645 [math-ph]]. 
[184] M. Petkovšek, H.S. Wilf and D. Zeilberger, A=B, (A.K. Peters, Wellesley, MA, 1996).

[185] M. Petkovšek and H. Zakrajšsek, in: Computer Algebra in Quantum Field Theory: Integration, Summation and Special Functions, eds. C. Schneider and J. Blümlein, Texts and Monographs in Symbolic Computation, (Springer, Wien, 2013), 259-284.

[186] J. Ablinger, J. Blümlein, S. Klein, and C. Schneider, Nucl. Phys. Proc. Suppl. 205-206 (2010) 110-115, [arXiv:1006.4797[math-ph]].

[187] C. Schneider, J. Phys. Conf. Ser. 523 (2014) 012037, [arXiv:1310.0160[cs.Sc]].

[188] H. Wilf and D. Zeilberger, Invent. Math. 108 (1992) 575-633.

[189] K. Wegschaider, Computer generated proofs of binomial multi-sum identities, Master Thesis, RISC, J. Kepler University, 1997.

[190] D. Zeilberger, J. Comput. Appl. Math. 32 (1990) 321-368.

[191] F. Chyzak, Discrete Math. 217 (2000) 115-134.

[192] J. Blümlein, M. Round, and C. Schneider, in: Advances in Computer Algebra - In Honour of Sergei Abramov's' 70th Birthday, WWCA 2016, Waterloo, Ontario, Canada, July 23-24, 2016, Series: Springer Proceedings in Mathematics \& Statistics, pp. 51-92, (Springer, Berlin, 2017), C. Schneider and E. Zima (Eds.), [arXiv: 1706.03677 [cs.SC]].

[193] J. Blümlein, A. Hasselhuhn, and C. Schneider, PoS (RADCOR2011) 032, [arXiv: 1202.4303 [math-ph]].

[194] C. Schneider, A. De Freitas, and J. Blümlein, PoS (LL2014) 017 [arXiv:1407.2537[cs.SC]].

[195] C. Schneider, J. Ablinger, J. Blümlein, and A. de Freitas, PoS (RADCOR2015) 060 [arXiv: 1601.01856 [cs.SC]].

[196] J. Ablinger, C. Schneider, A. Behring, J. Blümlein, and A. de Freitas, PoS (LL2016) 005 [arXiv: 1608.05376 $[$ cs.SC]].

[197] K. Symanzik, Commun. Math. Phys. 18 (1970) 227-246.

[198] C. G. Callan, Jr., Phys. Rev. D2 (1970) 1541-1547.

[199] A. Behring, I. Bierenbaum, J. Blümlein, A. De Freitas, S. Klein, and F. Wißbrock, Eur. Phys. J. C74 (2014), no. 9 3033, [arXiv: 1403.6356 [hep-ph]].

[200] M. Jezabek, Z. Phys. C56 (1992) 285-288.

[201] M. Skrzypek, Acta Phys. Polon. B23 (1992) 135-172.

[202] M. Przybycien, Acta Phys. Polon. B24 (1993) 1105-1114, [hep-th/9511029].

[203] J. Blümlein, S. Riemersma, and A. Vogt, Eur. Phys. J. C1 (1998) 255-259, [hep-ph/9611214].

[204] A. B. Arbuzov, Phys. Lett. B470 (1999) 252-258, [hep-ph/9908361].

[205] J. Blümlein and H. Kawamura, Nucl. Phys. B708 (2005) 467-510, [hep-ph/0409289].

[206] J. Blümlein and H. Kawamura, Eur. Phys. J. C51 (2007) 317-333, [hep-ph/0701019].

[207] J. Blümlein and H. Kawamura, Phys. Lett. B553 (2003) 242-250, [hep-ph/0211191].

[208] J. Blümlein, Prog. Part. Nucl. Phys. 69 (2013) 28-84, [arXiv:1208.6087[hep-ph]].

[209] J. Ablinger, PoS (LL2014) 019, [arXiv:1407.6180[cs.SC]].

[210] J. Ablinger, A Computer Algebra Toolbox for Harmonic Sums Related to Particle Physics, Diploma Thesis, JKU Linz, 2009, arXiv:1011.1176[math-ph].

[211] C. Duhr, H. Gangl, and J. R. Rhodes, JHEP 10 (2012) 075, [arXiv:1110.0458[math-ph]].

[212] C. Reutenauer, Free Lie Algebras (Calendron Press, Oxford, 1993).

[213] J. Blümlein, Comput. Phys. Commun. 159 (2004) 19-54, [hep-ph/0311046].

[214] M.E. Hoffman, J. Algebraic Combin. 11 (2000) 49-68 [arXiv:math/9907173[math.QA]].

[215] H. Hopf, Ann. of Math. 42 (1941) 22-52. 
[216] J. Milner and J. Moore, Ann. of Math. 81 (1965) 211-264.

[217] M.E. Sweedler, Hopf Algebras, (Benjamin, New York, 1969).

[218] D. Kreimer, Adv. Theor. Math. Phys. 2 (1998) 303-334, [q-alg/9707029].

[219] A. Connes and D. Kreimer, Commun. Math. Phys. 199 (1998) 203-242, [hep-th/9808042].

[220] A. Connes and D. Kreimer, Commun. Math. Phys. 210 (2000) 249-273, [hep-th/9912092].

[221] A. Connes and D. Kreimer, Commun. Math. Phys. 216 (2001) 215-241, [hep-th/0003188].

[222] R.C. Lyndon, Trans. Amer. Math. Soc. 77 (1954) 202-215; 78 (1955) 329-332.

[223] D.E. Radford, J. Algebra, 58 (1979) 432-454.

[224] E. Witt, Journ. Reine und Angew. Mathematik, 177 (1937) 152-160.

[225] E. Witt, Math. Zeitschr. 64 (1956) 195-216.

[226] J. Ablinger and C. Schneider, Ann. Comb. 22 (2018), in print https://doi.org/10.1007/s00026-018-0381-5, [arXiv:1510.03692 [cs.SC]].

[227] N. Nielsen, Handbuch der Theorie der Gammafunktion, (Teubner, Leipzig, 1906); reprinted by (Chelsea Publishing Company, Bronx, New York, 1965).

[228] E. Landau, Über die Grundlagen der Theorie der Fakultätenreihen, S.-Ber. math.-naturw. Kl. Bayerische Akad. Wiss. München, 36 (1906) 151-218.

[229] J. Blümlein, Clay Math. Proc. 12 (2010) 167-188, [arXiv:0901.0837[math-ph]].

[230] J. Blümlein, Comput. Phys. Commun. 180 (2009) 2218-2249, [arXiv:0901.3106[hep-ph]].

[231] J. Blümlein, Comput. Phys. Commun. 133 (2000) 76-104, [hep-ph/0003100].

[232] J. Blümlein and S.-O. Moch, Phys. Lett. B614 (2005) 53-61, [hep-ph/0503188].

[233] A.V. Kotikov and V.N. Velizhanin, Analytic continuation of the Mellin moments of deep inelastic structure functions, hep-ph/0501274.

[234] J. Blümlein and V. Ravindran, Nucl. Phys. B716 (2005) 128-172, [hep-ph/0501178].

[235] J. Blümlein and V. Ravindran, Nucl. Phys. B749 (2006) 1-24, [hep-ph/0604019].

[236] D. Maitre, Comput. Phys. Commun. 174 (2006) 222-240, [hep-ph/0507152].

[237] T. Gehrmann and E. Remiddi, Comput. Phys. Commun. 141 (2001) 296-312, [hep-ph/0107173].

[238] J. Ablinger, J. Blümlein, M. Round, and C. Schneider, PoS (RADCOR2017) 010 [arXiv:1712.08541[hep-th]].

[239] J. Henn, A. V. Smirnov, V. A. Smirnov, and M. Steinhauser, JHEP 01 (2017) 074, [arXiv:1611.07535[hep$\mathrm{ph}]]$.

[240] E.E. Kummer, J. Reine Angew. Math. (Crelle) 21 (1840) 74-90; 193-225; 328-371.

[241] H. Poincaré, Acta Math. 4 (1884) 201-312.

[242] J.A. Lappo-Danilevsky, Mémoirs sur la Théorie des Systèmes Différentielles Linéaires, (Chelsea Publ. Co, New York, 1953).

[243] K.T. Chen, Trans. A.M.S. 156 (3) (1971) 359-379.

[244] A.B. Goncharov, Math. Res. Lett. 5 (1998) 497-516.

[245] J. Blümlein, A. Hasselhuhn, S. Klein, and C. Schneider, Nucl. Phys. B866 (2013) 196-211, [arXiv: 1205.4184 [hep-ph]|.

[246] J. Ablinger, A. Behring, J. Blümlein, A. De Freitas, A. von Manteuffel, and C. Schneider, Nucl. Phys. B890 (2014) 48-151, [arXiv:1409.1135[hep-ph]].

[247] S. Weinzierl, Comput. Phys. Commun. 145 (2002) 357-370, [math-ph/0201011].

[248] S. Moch and P. Uwer, Comput. Phys. Commun. 174 (2006) 759-770, [math-ph/0508008].

[249] J. Vollinga and S. Weinzierl, Comput. Phys. Commun. 167 (2005) 177, [hep-ph/0410259]. 
[250] A. I. Davydychev and M. Yu. Kalmykov, Nucl. Phys. B699 (2004) 3-64, [hep-th/0303162].

[251] S. Weinzierl, J. Math. Phys. 45 (2004) 2656-2673, [hep-ph/0402131].

[252] J. Ablinger, J. Blümlein, A. De Freitas, A. Hasselhuhn, A. von Manteuffel, M. Round, and C. Schneider, Nucl. Phys. B885 (2014) 280-317, [arXiv:1405.4259[hep-ph]].

[253] A. Ablinger et al., DESY 15-112.

[254] J. Blümlein and C.G. Raab, in preparation.

[255] J. Ablinger, J. Blümlein, A. De Freitas, A. Hasselhuhn, C. Schneider, and F. Wißbrock, Nucl. Phys. B921 (2017) 585-688, [arXiv:1705.07030[hep-ph]].

[256] J. Ablinger, J. Blümlein, A. De Freitas, C. Schneider, and K. Schönwald, Nucl. Phys. B927 (2018) 339-367, [arXiv: 1711.06717 [hep-th]].

[257] J. Ablinger, J. Blümlein, A. De Freitas, A. Goedicke, C. Schneider, and K. Schönwald, arXiv: 1804.02226 [hep-ph].

[258] E. Remiddi and L. Tancredi, Nucl. Phys. B907 (2016) 400-444, [arXiv:1602.01481[hep-ph]].

[259] A. Ronveaux, ed., Heun's differential equations, (The Clarendon Press Oxford, Oxford, 1995).

[260] E. Imamoglu and M. van Hoeij, J. Symbolic Comput. 83 (2017) 245-271, [arXiv:1606.01576 [cs.SC]].

[261] K. Takeuchi, J. Fac. Sci, Univ. Tokyo, Sect. 1A 24 (1977) 201-272.

[262] S. Herfurtner, Math. Ann. 291 (1991) 319-342.

[263] H. Movasati and S. Reiter, Bull. Braz. Math Soc. 43 (2012) 423-442 [arXiv: 0902.0760[math.AG]].

[264] J. Blümlein, talks at: The 5th International Congress on Mathematical Software ZIB Berlin from July 11 to July 14, 2016, Session: Symbolic computation and elementary particle physics, https://www.risc.jku.at/conferences/ICMS2016/; and QCD@LHC2016, U. Zürich, August 22 to August 26, 2016, https://indico.cern.ch/event/516210/timetable/\#all.detailed.

[265] E. Remiddi and L. Tancredi, Nucl. Phys. B925 (2017) 212-251, [arXiv:1709.03622[hep-ph]].

[266] L. Adams and S. Weinzierl, Phys. Lett. B781 (2018) 270-278, [arXiv:1802.05020[hep-ph]].

[267] H.H. Chan and W. Zudilin, Mathematika 56 (2010) 107-117.

[268] D.J. Broadhurst, Eta quotients, Eichler integrals and L-series, talk at HMI Bonn, February 2018.

[269] L. Adams and S. Weinzierl, Communications in Number Theory and Physics in print [arXiv:1704.08895[hep$\mathrm{ph}]]$.

[270] J. Brödel, C. Duhr, F. Dulat, B. Penante, and L. Tancredi, Elliptic symbol calculus: from elliptic polylogarithms to iterated integrals of Eisenstein series, arXiv:1803.10256[hep-th].

[271] S. Abreu, R. Britto, C. Duhr, and E. Gardi, JHEP 12 (2017) 090, [arXiv:1704.07931[hep-th]].

[272] D. Hilbert, Grundzüge einer allgemeinen Theorie der linearen Integralgleichungen, (Teubner, Leipzig, 1912).

[273] R. de L. Kronig, J. Opt. Soc. Am. 12 (1926) 547-557.

[274] H.A. Kramers, Atti Cong. Intern. Fisici, (Transactions of Volta Centenary Congress) Como, 2 (1927).

[275] M. J. G. Veltman, Physica 29 (1963) 186-207.

[276] C. Neumann, Vorlesungen über Riemann's Theorie der Abel'schen Integrale, (Teubner, Leipzig, 1884), 2nd Edition.

[277] F. Brown and O. Schnetz, Duke Math. Journal 161 No. 10 (2012) 1817-1862. 